\title{
University of California, Berkeley
}

U.C. Berkeley Division of Biostatistics Working Paper Series

\section{Collaborative Targeted Maximum Likelihood For Time To Event Data}

\author{
Ori M. Stitelman* Mark J. van der Laan ${ }^{\dagger}$
}

*University of California - Berkeley, ostitelman@berkeley.edu

†University of California - Berkeley, laan@ berkeley.edu

This working paper is hosted by The Berkeley Electronic Press (bepress) and may not be commercially reproduced without the permission of the copyright holder.

http://biostats.bepress.com/ucbbiostat/paper260

Copyright (c) 2010 by the authors. 


\title{
Collaborative Targeted Maximum Likelihood For Time To Event Data
}

\author{
Ori M. Stitelman and Mark J. van der Laan
}

\begin{abstract}
Current methods used to analyze time to event data either, rely on highly parametric assumptions which result in biased estimates of parameters which are purely chosen out of convenience, or are highly unstable because they ignore the global constraints of the true model. By using Targeted Maximum Likelihood Estimation one may consistently estimate parameters which directly answer the statistical question of interest. Targeted Maximum Likelihood Estimators are substitution estimators, which rely on estimating the underlying distribution. However, unlike other substitution estimators, the underlying distribution is estimated specifically to reduce bias in the estimate of the parameter of interest. We will present here an extension of Targeted Maximum Likelihood Estimation for observational time to event data, the Collaborative Targeted Maximum Likelihood Estimator for the treatment specific survival curve. Through the use of a simulation study we will show that this method improves on commonly used methods in both robustness and efficiency. In fact, we will show that in certain situations the C-TMLE produces estimates whose mean square error is lower than the semi-parametric efficiency bound. Lastly, we will show that the bootstrap is able to produce valid 95 percent confidence intervals in sparse data situations, while influence curve based inference breaks down.
\end{abstract}




\section{Introduction}

It is common to want to quantify in observational data the effect of a treatment or exposure on the time it takes for an event to occur. In Moore and van der Laan 2009 they introduce the targeted maximum likelihood estimator for estimating the treatment specific survival curve in randomized clinical trials [2]. The targeted maximum likelihood estimator (TMLE) presented there improves upon common methods for analyzing time to event data in robustness, efficiency, and interpretability of parameter estimates. However, those methods may not be directly applied to observational data because it differs from randomized clinical trials in that the exposure/treatment is not set externally.

A 2009 paper by van der Laan and Gruber introduces a new class of estimators, the collaboratively double robust targeted maximum likelihood estimators (C-TMLE)[8]. These estimators are an extension of TMLE specific to the observational setting. The C-TMLE methods presented there provide estimates which, in many instances, are more efficient than standard TMLE and all other possible estimation techniques. In fact, the C-TMLE may produce super efficient estimates, or estimates which are more efficient than the semiparametric efficiency bound. Furthermore, the C-TMLE methodology produces well behaved estimates in situations where the parameter of interest is borderline identifiable. When exposure is not randomized there may be individuals with certain baseline characteristics which never experience a particular level of exposure. In other words, a certain set of baseline characteristics may be completely predictive of a particular treatment level. This phenomenon is termed a violation in the experimental treatment assumption (ETA) in Neugebauer and van der Laan 2005 [3]. Violations in ETA render the parameters of interest as presented in Moore and van der Laan unidentifiable. However, many times in finite samples certain parameters are weakly identifiable due to practical violations in the ETA assumption. Practical violations occur when a certain set of baseline covariates are almost completely predictive of a certain treatment within the sample. C-TMLE estimators address this issue and are able to produce stable estimates of borderline identifiable parameters. Thus, the C-TMLE methodology may be applied in the time to event setting to gain efficiency as well as produce estimates for weakly identifiable parameters of interest. van der Laan and Gruber present a general methodology for constructing C-TMLE estimators as well as apply the method to several basic data structures. We will extend those methods to time to event data and present a C-TMLE methodology for estimating the treatment specific survival curve. A simulation study will also be presented that illustrates the advantages of using C-TMLE versus other methods.

\section{Estimating Parameters In Coarsened Data Structures}

In this section we will briefly introduce the parameter estimation problem for a coarsened data structure. Then different methods for estimating parameters within this data structure will be introduced along with their shortcomings. Finally, we will discuss the TMLE 
methodology in general, and illustrate how the properties of targeted maximum likelihood estimates address the drawbacks seen in the other estimators.

Suppose one observes a censored data structure $O=\Phi(C, X)$ of the full data $X$ and censoring variable $C$ which has a probability distribution $P_{0}$. Let $\mathcal{M}$ be a semiparametric model for the probability distribution $P_{0}$. By assuming coarsening at random (CAR) the density factors as $d P_{0}(O)=Q_{0}(O) g_{0}(O \mid X)$, where $Q_{0}$ is the part of the density associated with the full data, $X$, and $g_{0}$ is the conditional distribution of the observed data, $O$, given the full data. $g_{0}$ includes both the censoring and treatment mechanism, which both act to coarsen the full data. The factorization of the density implies that the model $\mathcal{M}$ may be partitioned into a model $\mathcal{Q}$ for the full data distribution, $Q_{0}$, and model $\mathcal{G}$ for the censoring and treatment mechanism, $g_{0}$. So the probability distribution, $P_{0}$ may be indexed in the following way: $P_{Q_{0}, g_{0}}$. One is typically interested in estimating a parameter, $\Psi\left(P_{0}\right)$, which is a function of the true data generating distribution. More specifically, the parameter of interest is $\Psi\left(Q_{0}\right)$ which is a function of the true full data generating distribution absent coarsening.

Many methods have been developed to estimate $\Psi\left(Q_{0}\right)$. Traditional maximum likelihood estimation methods approach this problem by producing a substitution estimator $\Psi(\hat{Q})$, where maximum likelihood is used to estimate $\hat{Q}$. Since the model $\mathcal{M}$ can be very large, this would require sieve-based (data adaptive) maximum likelihood estimation (i.e., loss-based machine learning), involving fine-tuning of the amount of smoothing used. ${ }^{1}$ If $\Psi\left(Q_{0}\right)$ denotes a causal effect, this estimator is referred to as the G-computation estimator (G-comp). Such methods typically produce overly biased estimates of the parameter of interest since the estimate of $Q_{0}$ is at best created with concern for the bias variance trade off of the entire density rather than for the parameter of interest. However, the G-comp estimator does respect the global constraints of the model by acknowledging that the parameter of interest is is a function of $Q_{0}, \Psi\left(Q_{0}\right)$. Thus, the estimate, $\hat{\psi}^{\text {gcomp }}$, is a substitution of a maximum likelihood estimator, $\hat{Q}$ in the model $\mathcal{Q}$, into the parameter mapping $\Psi()$. In addition, by using state of the art loss (log-likelihood) based learning, it provides low-variance estimators, inspired by the efficient parametric maximum likelihood estimation.

An alternative method for estimating $\Psi\left(Q_{0}\right)$ is the inverse probability of treatment based approach (IPW). IPW estimators solve an estimating equation in order to yield estimates, $\hat{\psi}^{I P W}$, of the parameter of interest. $\hat{\psi}^{I P W}$ are consistent estimates of $\Psi\left(Q_{0}\right)$ when one estimates the $g$ part of the likelihood consistently. However, IPW estimates are highly unstable for two reasons. First, IPW estimates do not solve the efficeint influence curve estimating equation. Second, they do not respect the global restraints of a proper

\footnotetext{
${ }^{1}$ A detailed account of loss based learning and cross validation may be seen in van der Laan and Dudoit 2003 [6]. In addition, van der Laan, Keles and Dudoit focus specifically on likelihood-based cross-validation and illustrate its asymptotic optimality in their 2004 article [7]. van der Laan, Polley, and Hubbard rely on the results of the previous two papers in their 2007 article which presents the Super Learner methodology, a data-adaptive learning technique which utilizes likelihood based cross-validation[9].
} 
model by being a substitution estimator. As a result IPW estimates are highly variable and act erratically in finite samples.

Another method for estimating $\Psi\left(Q_{0}\right)$ is augmented inverse probability weighted estimators (AIPW). Robins and Rotntizky proposed this general estimating equation based approach in 1992 which constructs estimators, $\hat{\psi}^{A I P W}$, that solve the efficient influence curve estimating equation [5]. The estimates produced by these methods are referred to as double robust because they are consistent when either the $Q$ or the $g$ part of the likelihood are estimated consistently. Furthermore, they also improve on the standard inverse probability weighted estimators in terms of efficiency since they solve the efficient influence curve equation; thus, they are locally efficient. However, like the IPW estimators, the AIPW estimators are not substitution estimators and may also be unstable. For a general treatment of these methods see Robins and van der Laan 2003 [10].

van der Laan and Rubin in their 2006 paper introduce a new class of estimators, the targeted maximum likelihood estimators (TMLE) [11]. The TMLE methodology developed in that paper is a two stage process which results in a substitution estimator. In the first stage an initial estimate, $\hat{Q}^{0}$, of $Q_{0}$ is obtained, using loss-based learning. In the second stage the first stage estimate is fluctuated to reduce bias in the estimate of the parameter of interest. The bias is reduced by insuring that the efficient influence curve equation is solved by the targeted maximum likelihood solution $\hat{Q}^{*}$. This is achieved by finding the targeted maximum likelihood estimate of $Q_{0}, \hat{Q}_{g}^{*}$, with a parametric fluctuation model whose score at the initial estimator (i.e., at zero fluctuation) equals or includes the efficient influence curve of the parameter of interest. This may be done by specifying a univariate regression model for the outcome of interest on a covariate, $h\left(\hat{Q}^{0}, \hat{g}\right)$, specifically chosen to yield the appropriate score while using the initial estimator of $\hat{Q}^{0}$ as an offset. $h\left(\hat{Q}^{0}, \hat{g}\right)$ is a function of $\hat{g}$ and thus the second stage requires an estimate $\hat{g}$ of $g_{0}$. The coefficient $\epsilon$ in front of the clever covariate, $h\left(\hat{Q}^{0}, \hat{g}\right)$, is then estimated using standard parametric maximum likelihood. This is known as the first targeted maximum likelihood step and yields $\hat{Q}^{1}$, the first step targeted maximum likelihood estimate of $Q_{0}$. The targeted maximum likelihood step is then iterated using $\hat{Q}^{1}$ as the initial estimator of $Q_{0}$ and the estimate of $g_{0}$ remains unchanged. This process is iterated until $\epsilon$ converges to zero, resulting in the targeted maximum likelihood estimate of $Q_{0}$, or $\hat{Q}_{g}^{*} . \Psi\left(\hat{Q}_{g}^{*}\right)$ is the targeted maximum likelihood estimate of the parameter $\Psi\left(Q_{0}\right)$. Note, that $\hat{Q}_{g}^{*}$ is indexed by the treatment and censoring mechanisms, g. This is because unlike the G-computation estimators above, the TMLE, through $h$, makes use of the fact that the observed data is generated according to a censored data structure as dictated by the efficient influence curve for $\Psi\left(Q_{0}\right)$.

Like the AIPW estimators the TMLE estimates are double robust and locally efficient; however, the TMLE methedology improves on the AIPW approach that also solves the efficient influence curve equation in four major ways:

1. The TMLE respects the global constraint of the model and the AIPW estimate does not. Since the TMLE is a substitution estimator and maps the targeted maximum 
likelihood estimate $\hat{P}^{*}$ of $P_{0}$ into the parameter mapping $\Psi()$, it respects knowledge of the model. By solving an estimating equation, AIPW estimators, in most instances, do not acknowledge that one is estimating a parameter which is a function of the underlying data generating distribution. One situation where this issue is glaringly obvious is when AIPW estimators can return estimates which are out of the natural bounds of the problem, as is the case when one is estimating a probability that must fall between 0 and 1 . However, the implications of respecting the knowledge of the model are more subtle than just returning estimates which are out of the natural bounds of the parameter and this advantage contributes to finite sample gains in efficiency which will be displayed in the simulation results presented below, and are particularly strong in the context of sparsity w.r.t. the target parameter (i.e., the sample contains sparse information about the parameter of interest).

2. The log-likelihood of $\hat{Q}_{g}^{*}$, or targeted maximum likelihood, is a direct measure of fit upon which to choose among different estimates of $g$ and $Q$. Prior to TMLE, estimators which relied on estimates of $Q$ and $g$ to identify $P_{Q_{0}, g_{0}}$ distinguished between different estimates based on how well they do for prediction by using a loss function for the global density. Whereas, the TMLE methedology uses the targeted maximum likelihood to choose among different estimates based on how they help in estimating the parameter of interest, $\Psi\left(Q_{0}\right)$. This advantage is particularly striking in the typical context that there are excellent fits of $g_{0}$ itself, resulting in unstable inverse of probability of censoring weights, that truly harm the bias reduction effort for the target parameter of $Q_{0}$.

3. The TMLE can produce estimates when the efficient influence curve may not be written as an estimating function in terms of the parameter of interest, $\psi$. The AIPW estimate requires that the efficient influence curve be represented as an estimating function. The log-rank parameter, as presented in Moore and van der Laan 2009, is an example of a parameter whose efficient influence curve may not be written as an estimating function and thus can be estimated directly through TMLE but not directly by AIPW estimators.

4. The TMLE does not have multiple solutions. Since AIPW estimates are solutions to estimating equations they may have multiple solutions. The estimating equation itself provides no criteria upon which to choose between the multiple solutions. TMLE does not suffer from multiple solutions since it is a loss-based learning approach, e.g., maximizing the log-likelihood fit, involving second stage extra fitting along a targetparameter specific parametric fluctuation model, which just happens to also solve the efficient influence curve estimating equation in the probability distribution.

How these advantages, specifically the first two, actually effect the properties of the estimates produced by the TMLE methedology will be explored in the following sections and 
be quantified in comparison to other methods through a simulation study presented in sections 12 through 14.

\section{Collaborative Targeted Maximum Likelihood Estimation In General}

In their original paper on C-TMLE van der Laan and Gruber introduce a new and stronger type of double robustness entitled collaborative double robustness. The collaboratively double robustness property of an (targeted ML) estimator $\hat{Q}_{\hat{g}}$ of $Q_{0}$ only requires that the estimate of $g_{0}, \hat{g}$, account for variables that effect $Q_{0}$ and were not fully accounted for in the initial $\hat{Q}$. Thus the property does not require that either the estimates of $Q$ or $g$ are consistent, but rather is concerned with reducing the distance between, $\hat{Q}_{\hat{g}}$ and $Q_{0}$, and, $\hat{g}$ and $g_{0}$, such that the resulting estimator of $\Psi\left(Q_{0}\right)$ is unbiased. So if $\hat{Q}$ does a very good job estimating $Q_{0}$, very little adjustment is necessary through the estimate of $g$; on the other hand, if $\hat{Q}$ is a poor estimate of $Q_{0}$, the estimate $\hat{g}$ will have to do a better job of estimating $g_{0}$ with respect to those variables that effect $Q_{0}$.

The C-TMLE methodology, introduced by van der Laan and Gruber, is an extension of TMLE that takes advantage of the collaborative doubly robust property of those estimators by constructing $\hat{g}$ in collaboration with $\hat{Q}$. C-TMLE uses the log-likelihood as a loss function to choose from a sequence of $\mathrm{K}$ targeted maximum likelihood estimates, $\hat{Q}^{k *}$, indexed by initial estimates of $Q_{0}$ and $g_{0}$. In their paper, van der Laan and Gruber provide a framework for generating C-TMLEs which we will briefly outline now:

1. Create $\hat{Q}$, an initial estimator of $Q_{0}$.

2. Generate a sequence of estimates of $g_{0}: \hat{g}^{0}, \hat{g}^{1}, \ldots, \hat{g}^{K-1}, \hat{g}^{K}$. Where $\hat{g}^{0}$ is the least data adaptive estimate and $\hat{g}^{K}$ is the most data adaptive estimate of $g_{0}$.

3. Generate the initial TMLE estimate, $\hat{Q}^{0 *}$, indexed by $\hat{Q}$ and $\hat{g}^{0}$.

4. Generate a sequence of TMLE estimates: $\hat{Q}^{0 *}, \hat{Q}^{1 *} \ldots, \hat{Q}^{K-1 *}, \hat{Q}^{K *}$ indexed by $\hat{g}^{k}$; where each estimate has a larger log-likelihood than the last. This monotonicity is insured by adding an additional clever covariate to estimate $\hat{Q}^{k *}$ each time the loglikelihood does not increase within the same clever covariate just by virtue of the more data adaptive estimate of $g$. Whenever a new clever covariate is added for $\hat{Q}^{k *}$, $\hat{Q}^{k-1 *}$ is used as the initial estimate in the TMLE algorithm.

5. Finally, choose among the sequence of TMLE estimates using loss based crossvalidation with log-likelihood loss.

One adjustment to the above methodology, suggested by van der Laan and Gruber, is to use a penalized loss function when parameters are borderline identifiable. This is a very 
important consideration in observational studies and the issue of choosing an appropriate penalty is addressed in section 6 .

The C-TMLE has two distinct advantages over the TMLE methodology:

1. C-TMLE may be used to produce stable estimates of borderline identifiable parameters while TMLE (or any of the estimating equation methods discussed above) breakdown in these situations. The reason many parameters are not identifiable, or are borderline identifiable, is due to violations in ETA, where a certain level of a covariate or group of covariates is completely predictive of treatment/exposure. In these situations, where sparsity of the data is an issue, C-TMLE is able to weigh the bias-variance trade off of adjusting for certain covariates in estimating these weakly identifiable parameters. Thus, C-TMLE only adjusts for covariates in estimating $g_{0}$ when they are beneficial to the estimate of the parameter of interest and selects against adjusting for covariates which are detrimental to the final estimate of $\Psi\left(Q_{0}\right)$, weighing both bias and variance. All other methods estimate $g_{0}$ using a loss function for prediction, or a priori specifying a model, ignoring the effect adjusting for certain covariates has on the final estimate of the parameter of interest.

2. C-TMLE estimates in many situations are more efficient in finite samples than TMLE estimates. In fact, in some situations they are super-efficient and have a variance smaller than the semi-parametric efficiency bound. This is also a consequence of the collaborative double robustness of these estimates. In situations where the initial estimate, $\hat{Q}$ is a very good estimate of $Q_{0}$ in the targeted sense little adjustment is needed from the estimate of $g_{0}$. The more one adjusts for $g$ the larger the variability of the final estimate of the parameter of interest and thus not adjusting much in $g$ when one doesn't have to provides estimates with smaller variance. In some rare situations C-TMLE estimates have also been shown to be asymptotically super efficient: for example, if the initial estimator $\hat{Q}$ is a MLE for a correctly specified parametric model.

The C-TMLE estimates exhibit all of the advantages of the TMLE estimates discussed in the previous section as well as these two major advantages presented here. The advantages of C-TMLE estimators are particularly useful in observational studies, where practical violations in the ETA assumption are a concern; however, in studies where treatment is randomized and one attempts to gain efficiency by estimating $g$ C-TMLE estimators are also appropriate because they address the bias variance trade off of adjusting for particular variables. $^{2}$ Thus, implementation of the C-TMLE methods even for randomized treatments will help insure that one does not adjust in $g$ for the covariates in a way that hinders the estimate of the parameter of interest. In the following sections we will use a specific coarsened data structure, time to event data, to exhibit the advantages of C-TMLE.

\footnotetext{
${ }^{2}$ For a general account of how estimating the nuisance parameter mechanism even when it is known contributes to a gain in efficiency see van der Laan and Robins (2003), Section 2.3.7.
} 


\section{Data Structure}

Time to event analyses typically intend to assess the causal effect of a particular exposure, $A$, on the time, $T$, it takes for an event to occur. However, many times subjects are lost to follow up and as a result $T$ is not observed for all individuals. Individuals for whom the event is not observed are referred to as censored. This type of censoring is called right censoring since chronological time is arbitrarily chosen to progress from left to right and at some point in time, $C$, an individual is no longer observed; thus, all time points to the right of $C$ are censored for that individual. Some baseline covariates, $W$, are also recorded for each individual.

The observed data consists of $n$ i.i.d copies of, $O=(A, W, \tilde{T}, \Delta)$. Where $A$, is a binary variable, which quantifies the level of exposure/treatment a particular subject experiences, $W$ is a vector of baseline covariates, $\tilde{T}$ is the last time point at which a subject is observed, and $\Delta$ indicates whether the event occurred at $\tilde{T}$. So $\tilde{T}=\min (T, C)$ and $\Delta=I(T \leq C)$, where $C$ is the time at which a subject is censored. If the event is observed, $\Delta=1$, and $C$ is set equal to $\infty$. Alternatively if the event is not observed, $\Delta=0$, and $T$ is set equal to $\infty$. This formulation of the data structure is termed the short form of survival data and includes a row in the data set for each subject observed. Table 1 presents as an example four observations with selected baseline covariates from a sample HIV data set in their short form.

\begin{tabular}{rrrrrrr}
\hline Subject & $\tilde{T}$ & $\Delta$ & A & Sex & MSM & Baseline CD4 \\
\hline 1 & 6 & 1 & 1 & 1 & 1 & 439 \\
2 & 5 & 0 & 1 & 1 & 0 & 71 \\
3 & 5 & 0 & 0 & 1 & 0 & 118 \\
4 & 3 & 1 & 1 & 0 & 0 & 106 \\
\hline
\end{tabular}

Table 1: Example of sample HIV data in short form.

Alternatively, one may represent the same data in its long form. In this form the data set includes a row for each observation for each time the subject is observed up until it is either censored or experiences the event of interest. In order to represent the data in its long form two additional random variables must be introduced $N_{1}(t)$ and $N_{2}(t)$, where the first is an indicator that the event happens at time $t$, and the second is an indicator that a subject is censored at time t. Thus, subject 1 would have six rows in the data set in its long form and $N_{1}(t)$ will equal zero in every row except for the last row at $\mathrm{t}=6$ and $N_{2}(t)$ will equal zero in each row. Table 2 displays the same four observations from Table 1 in their long form. The baseline values, A and W, are repeated at each time point for each subject. Presenting the data in its long form allows for more flexibility and is essential for representing a general time dependent process (e.g., when dealing with time dependent covariates, variables whose value change over time, and/or counting processes 
that can jump more than once). In fact, it will be shown below that estimating TMLEs involves construction of a time dependent covariate, since $h(\hat{Q}, \hat{g})$ is a function of $t, A$, and $W$ and can be written as $h(t, A, W)$. So the data in its long form is $\mathrm{n}$ i.i.d. copies of $O=\left(A, W, N_{1}(t), N_{2}(t): t=1, \ldots, \tilde{T}\right) \sim p_{0}$, where $p_{0}$ denotes the density of the observed data, $O$.

\begin{tabular}{rrrrrrrr}
\hline Subject & $t$ & $N_{1}(t)$ & $N_{2}(t)$ & A & Sex & MSM & Baseline CD4 \\
\hline 1 & 1 & 0 & 0 & 1 & 1 & 1 & 439 \\
1 & 2 & 0 & 0 & 1 & 1 & 1 & 439 \\
1 & 3 & 0 & 0 & 1 & 1 & 1 & 439 \\
1 & 4 & 0 & 0 & 1 & 1 & 1 & 439 \\
1 & 5 & 0 & 0 & 1 & 1 & 1 & 439 \\
1 & 6 & 1 & 0 & 1 & 1 & 1 & 439 \\
2 & 1 & 0 & 0 & 1 & 1 & 0 & 71 \\
2 & 2 & 0 & 0 & 1 & 1 & 0 & 71 \\
2 & 3 & 0 & 0 & 1 & 1 & 0 & 71 \\
2 & 4 & 0 & 0 & 1 & 1 & 0 & 71 \\
2 & 5 & 0 & 1 & 1 & 1 & 0 & 71 \\
3 & 1 & 0 & 0 & 0 & 1 & 0 & 118 \\
3 & 2 & 0 & 0 & 0 & 1 & 0 & 118 \\
3 & 3 & 0 & 0 & 0 & 1 & 0 & 118 \\
3 & 4 & 0 & 0 & 0 & 1 & 0 & 118 \\
3 & 5 & 0 & 1 & 0 & 1 & 0 & 118 \\
4 & 1 & 0 & 0 & 1 & 0 & 0 & 106 \\
4 & 2 & 0 & 0 & 1 & 0 & 0 & 106 \\
4 & 3 & 1 & 0 & 1 & 0 & 0 & 106 \\
\hline
\end{tabular}

Table 2: Example of sample HIV data in long form.

\section{Causal Assumptions and Factorization Of Observed Data Likelihood}

The data structure presented in the preceding section implies little about the causal structure of the underlying mechanisms which produce the observed data. The temporal structure which is implied by the fact that $A$ and $W$ are measured at baseline and then some time in the future, $\mathrm{T}$, an event occurs is clear from the observed data structure; however, no assumptions have been made as to how $A$ effects the outcome, $T$. Does $A$ directly effect $T$ ? Does $A$ effect $W$ which then effects $T$ ? Does $A$ effect $T$ through $W$ as well as directly? Does $A$ even cause $W$ or does $W$ cause $A$ ? In fact, there are countless numbers of ways 
which the observed random variables could have caused or been caused by each other. In order to quantify, or even define, the causal effect of $A$ on the outcome $T$ causal assumptions are necessary. Causal graphs succinctly lay out the causal assumptions necessary to define a causal effect as well as provide much of the information needed to determine if the effect is estimable from the observed data. For a detailed account of causal graph theory see Pearl 2008 [4]. One constructs a causal graph based on the cues provided from the world in which the observed phenomenon are occurring, whether it be based on subject knowledge or constraints imposed by how an experiment is designed. ${ }^{3}$ Such issues are out of the scope of this paper and we will just assume that the causal graph presented in Figure 1 depicts the data generating mechanisms for the observed data. Exogenous error nodes, whose joint distribution is such that there are no unblocked backdoor paths from $A$ and the $N_{1}(t)$ nodes to any of the $N_{2}(t)$ nodes of the event process, are suppressed in the causal graph, as is typically done in the literature. Thus, each node is defined in the causal graph as a function of its ancestors and an exogenous error node which is not depicted in the graph.

The causal graph depicted in Figure 1 presents a common structure for which one may be interested in the causal effect of $A$ on the time until an event occurs. This causal structure corresponds with the causal structure examined in Moore and van der Laan 2009, except for the fact that $W$ now is a direct cause of $A$, indicated by an arrow from $W$ to $A$. This change is due to the fact that $A$ is not externally set as in a randomly controlled experiment, but rather, is free to be whatever it would be as naturally observed. Thus, other variables, $W$, effect what level of $A$ a subject experiences.

The causal graph allows one to make necessary assumptions typically stated to make causal parameters estimable. The consistency assumption, and coarsening at random assumption are made directly by the causal graph. The consistency assumption states that the observed outcome is the counterfactual outcome under the intervention actually observed. This assumption is a direct consequence of defining each node in the causal graph as a function of its ancestors and exogenous error nodes, and defining the observed data $O$ as the observed nodes generated by the nonparametric structural equation model represented by the causal graph. The coarsening at random assumption (CAR) states that the coarsening mechanism is only a function of the full data, the data in which you would have seen all counterfactuals, through the observed data. For the treatment variable this assumption is sometimes referred to as the randomization assumption or no unmeasured confounders assumption. This is directly implied by the causal graph since the treatment and censoring process variables only have arrows into them from their observed ancestors and no other nodes. This may also be confirmed by applying the no unblocked backdoor path criteria presented by Pearl [4].

\footnotetext{
${ }^{3}$ Pearl 2008 discusses algorithms which can search over observed data and provide a set of causal graphs which are consistent with that data. This use of causal graph theory is not consistent with our goal. For our purposes the causal graph is used to succinctly impose causal assumptions which we are able to make based off of knowledge of the mechanisms at work.
} 


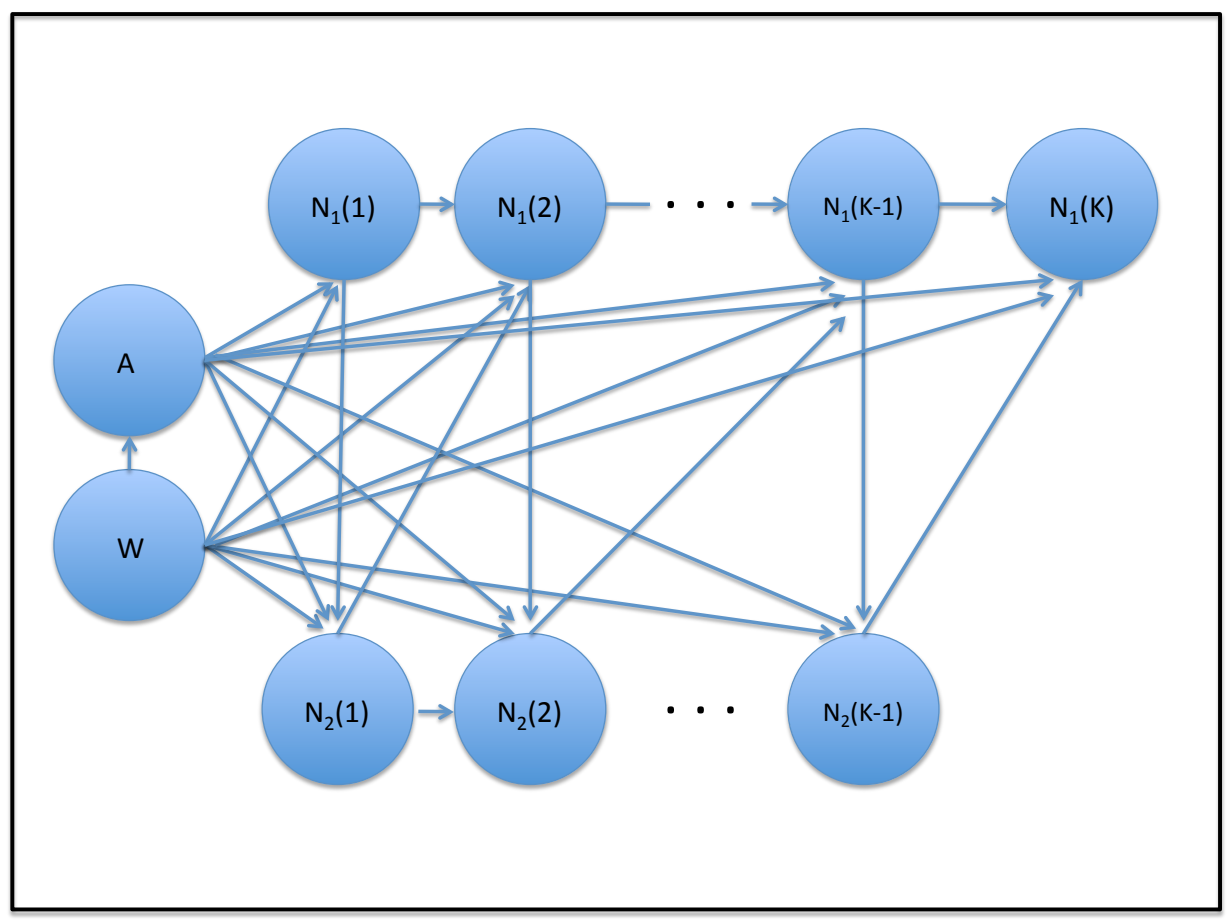

Figure 1: Causal graph depicting mechanisms which generate observed data. 
The causal graph presented in Figure 1 suggests the following orthogonal factorization of the likelihood of the observed data:

$$
\begin{aligned}
\mathcal{L}(O)= & \overbrace{P(W)}^{Q_{10}} \overbrace{P(A \mid W)}^{g_{10}} \prod_{t=1}^{K} \overbrace{P\left(N_{1}(t) \mid N_{1}(t-1), N_{2}(t-1), A, W\right)}^{Q_{20}} \\
& \underbrace{P\left(N_{2}(t) \mid N_{1}(t), N_{2}(t-1), A, W\right)}_{g_{20}}
\end{aligned}
$$

Thus, the likelihood factorizes as the general censored data structure presented in section 2 into a portion corresponding to the full data distribution, $Q_{0}$, and a portion corresponding to the censoring and treatment mechanism, $g_{0}$. $Q_{0}$ is composed of the baseline covariate distribtion, $Q_{10}(W)$ and $Q_{20}(t, A, W) \equiv E\left(d N_{1}(t) \mid N_{1}(t-1)=0, N_{2}(t-1)=0, A, W\right)$, the intensity of the event counting process given the treatment, $A$, and the baseline covariates, $W$, conditioning on "no event yet". $g_{0}$ is further factorized into the treatment mechanism, $g_{10}(A, W)$, and censoring mechanism, $g_{20}(t, A, W) \equiv E\left(d N_{2}(t) \mid N_{1}(t)=0, N_{2}(t-1)=\right.$ $0, A, W)$, which is the intensity of the censoring process given treatment, $A$, and the baseline covariates, $W$, conditioning on "no event yet". Let's also define $S_{0}\left(t_{k} \mid A, W\right)=\operatorname{Pr}(T>$ $\left.t_{k} \mid A, W\right)$ which is the conditional survival of the event of interest corresponding to the intensity of the event process, $Q_{20}(t, A, W)$, under the coarsening at random assumption:

$$
S_{0}\left(t_{k} \mid A, W\right)=\prod_{t=1}^{t_{k}}\left[1-Q_{20}(t, A, W)\right] .
$$

Note that $Q_{20}(t, A, W)$ is the conditional hazard of $T$ at $t$, given $A, W$, under CAR, which holds if $T$ and $C$ are conditionally independent, given $A, W$ (and was implied by our causal graph).

\section{Parameter of Interest}

Now that the likelihood of the data generating distribution has been introduced, parameters of interest which are functions of the data generating distribution, $\Psi\left(p_{0}\right)$, may be defined. Defining the parameter of interest as a mapping from the data generating distribution allows one to estimate exactly the feature of the data generating distribution that they are interested in. The treatment specific survival curve at a particular time point, $t_{k}$, is a simple example of this type of parameter, $\operatorname{Pr}\left(T_{a}>t_{k}\right)$. Where $T_{a}$ is the event time, $T$, one would have observed had an individual been set to treatment level a. This type of intervention in the real world may now be "observed" in our model as an intervention on the causal graph which sets an individual to level $A=a$. Since we are dealing with time to event data it is also necessary to intervene on the censoring mechanism at each time by setting the individual to uncensored, or $N_{2}(t)=0$. These interventions on the nodes associated with the $g$ part of the likelihood in the causal graph allow one to assess the distribution of 
the outcome had individuals been set to particular levels of treatment/exposure which are of interest in a world without censoring.

By intervening on the causal graph in Figure 1 by setting treatment A equal to the desired treatment and setting $N_{2}(t)$ to no censoring one may "observe" the event process under the desired treatment and without censoring. This outcome under the proposed intervention is known as the counterfactual outcome. Based on the causal assumptions imposed by the causal graph we can now write the treatment specific survival probability in terms of the data generating distribution, $p_{0}$ :

$$
\Psi_{a}\left(p_{0}\right)\left(t_{k}\right)=\operatorname{Pr}\left(T_{a}>t_{k}\right)=E_{W}\left(S_{0}\left(t_{k} \mid A=a, W\right)\right) .
$$

Parameters which combine $\Psi_{1}\left(p_{0}\right)\left(t_{k}\right)$ and $\Psi_{0}\left(p_{0}\right)\left(t_{k}\right)$ allow one to quantify the effect of a A on survival, T. Three examples of these types of parameters are the marginal additive difference in the probability of survival, the relative risk of survival, and the log of the marginal relative log hazard of survival:

$$
\begin{aligned}
& \Psi_{R D}\left(p_{0}\right)\left(t_{k}\right)=\Psi_{1}\left(p_{0}\right)\left(t_{k}\right)-\Psi_{0}\left(p_{0}\right)\left(t_{k}\right), \\
& \Psi_{R R}\left(p_{0}\right)\left(t_{k}\right)=\frac{\Psi_{1}\left(p_{0}\right)\left(t_{k}\right)}{\Psi_{0}\left(p_{0}\right)\left(t_{k}\right)}, \\
& \Psi_{R H}\left(p_{0}\right)\left(t_{k}\right)=\log \left(\frac{\log \left(\Psi_{1}\left(p_{0}\right)\left(t_{k}\right)\right)}{\log \left(\Psi_{0}\left(p_{0}\right)\left(t_{k}\right)\right)}\right) .
\end{aligned}
$$

Parameters which average these quantities over a set of time points may also be of interest. For the simulation study presented below we will just focus on the treatment specific survival curve at a particular time point.

\section{Alternative Methods For Estimating The Treatment Spe- cific Survival}

In this section we will briefly introduce the G-comp, IPW, and AIPW estimators for the treatment specific survival curve. ${ }^{4}$ These three alternatives will then be compared to the TMLE/C-TMLE estimates in the simulation study presented in Section 11.

\footnotetext{
${ }^{4}$ It is also common in analyses that intend to test weather or not a treatment or exposure, A, has an effect on a particular time to event outcome, to a priori specify a parametric model of the hazard, $Q_{20}\left(N_{1}(t), A, W\right)$, and to test if the coefficient on A in the specified model is different from zero. For continuous time a cox proportional hazard model is typically employed and for discrete time outcomes a logistic failure time model is used. Such methods are not examined here because they rely on highly parametric assumptions which typically result in biased estimates of parameters which are purely chosen out of convenience.
} 
G-computation estimators are one class of estimators for estimating the mean counterfactual outcome as expressed in the parameters proposed above. G-computation estimators are substitution estimators and thus require an estimate of the data generating distribution. In particular, the estimators require an estimate of the marginal distribution of $\mathrm{W}$, $Q_{10}(W)$, and the conditional hazard, which is $Q_{20}(t, A, W)$ under an assumption of coarsening at random. G-computation estimators of the parameter of interest, $\Psi$, are consistent when these distributions are estimated consistently. The marginal distribution of $\mathrm{W}$ is estimated non-parametrically by the empirical distribution of the baseline covariates in the sample. Super Learner, a data-adaptive machine learning algorithm which uses likelihoodbased cross validation to choose among a library of different learners is a good method for estimating $Q_{20}$ and subsequently obtaining $\hat{S}\left(t_{k} \mid A, W\right)$ [9]. The G-Computation estimate of the treatment specific survival, $\Psi_{a}\left(p_{0}\right)\left(t_{k}\right)$, may be expressed as:

$$
\hat{\psi}_{a}^{g-c o m p}=\frac{1}{n} \sum_{i=1}^{n} \hat{S}\left(t_{k} \mid A=a, W_{i}\right) .
$$

The IPW method for estimating the treatment specific survival curve only relies on estimates of the $g$ part of the likelihood. This estimator is an estimating equation based estimator and takes the following form:

$$
\hat{\psi}_{a}^{I P W}=\frac{1}{n} \sum_{i=1}^{n} \frac{I\left(T>t_{k}\right) I\left(C>t_{k}\right) I(A=a)}{\hat{g}_{1}(a \mid W) \prod_{i=1}^{t_{-}}\left[1-\hat{g}_{20}(i \mid A, W)\right]} .
$$

The AIPW estimator is a double robust estimator which solves the efficient influence curve based estimating equation. Thus, this estimator requires both estimates of the $Q$ and $g$ factors of the likelihood. The efficient influence curve for the treatment specific survival curve for this observed data structure is:

$$
\begin{aligned}
D_{a}^{*}\left(p_{0}\right)= & \sum_{t \leq t_{k}} h_{a}(t, A, W)\left[I(\tilde{T}=t, \Delta=1)-I(\tilde{T} \geq t) Q_{20}(t, A=a, W)\right] \\
& +S_{0}\left(t_{k} \mid A=1, W\right)+\Psi_{a}\left(p_{0}\right)\left(t_{k}\right),
\end{aligned}
$$

where

$$
h_{a}(t, A, W)=-\frac{I(A=a)}{g_{10}(A=a \mid W) \prod_{i=1}^{t}\left[1-g_{20}(i \mid A, W)\right]} \frac{S_{0}\left(t_{k} \mid A, W\right)}{S_{0}(t \mid A, W)} I\left(t \leq t_{k}\right)
$$

Hubbard et al. develop the one-step AIPW estimator which solves the efficient influence curve estimating equation in their 1999 paper [1]. The resulting AIPW estimate is:

$$
\hat{\psi}_{a}^{A I P W}=\frac{1}{n} \sum_{i=1}^{n} \sum_{t \leq t_{k}} \hat{h}_{a}(t, A, W)\left[I(\tilde{T}=t, \Delta=1)-I(\tilde{T} \geq t) \hat{Q}_{2}\left(N_{1}(t)=1, A=a, W\right)\right]+\hat{S}\left(t_{k} \mid A=1, W\right)
$$


where $\hat{h}_{a}(t, A, W)$ is $h_{a}(t, A, W)$ with estimates $\hat{g}_{1}, \hat{g}_{2}$, and $\hat{S}$ are substituted for $g_{10}, g_{20}$ and $S_{0}$.

\section{Targeted Maximum Likelihood Estimation Of Treatment Specific Survival}

Moore et al. (2009) introduced the TMLE estimate for the treatment specific survival curve, for the data structure of interest here. The TMLE requires an initial fit $\hat{p}^{0}$ of $p_{0}$, consisting of initial estimates $\hat{Q}_{1}, \hat{Q}_{2}^{0}(t, A, W), \hat{g}_{1}(A, W)$, and $\hat{g}_{2}(t, A, W)$. The 0 on the top indicates that it is the estimate prior to any iterations of the targeted maximum likelihood process. $\hat{Q}_{1}$ is the empirical distribution of observed $W$ and the other three estimates should be obtained using data adaptive methods. By updating the initial estimate $\hat{Q}_{2}^{0}(t, A, W)$ of the conditional hazard of $T$, by adding the term $\epsilon h$ on the logit-scale, such that the score for the likelihood of $O$ at $\epsilon=0$ is equal to the efficient influence curve (equation 9), a distribution targeted toward the parameter of interest may be constructed. In practice this may be done by implementing a standard univariate logistic regression of the outcome on $h(t, A, W)$ using the initial estimate $\hat{Q}_{2}^{0}(t, A, W)$ as an offset. The covariate $h(t, A, W)$ is presented in equation 10.

Since $h_{a}(t, A, W)$ is a function of $Q_{20}(t, A, W)$ it is necessary to iterate the updates $\mathrm{K}$ times, updating $\hat{Q}_{2}^{k}(t, A, W)$ until $\hat{\epsilon}$ converges to zero. The resulting estimate after convergence, $Q_{2}^{*}$, is the conditional hazard targeted toward estimating the parameter of interest, which combined with the empirical distribution of $W$ yields the portion of the density of $O$ needed for evaluation of the target parameter. Finally, the targeted maximum likelihood estimate of $\Psi\left(\hat{p}^{*}\right)\left(t_{k}\right)$ is the substitution estimator based on the targeted density $\hat{p}^{*}:$

$$
\hat{\psi}_{a}^{T M L E}=\frac{1}{n} \sum_{i=1}^{n} \hat{S}_{a}^{*}\left(t_{k} \mid 1, W_{i}\right),
$$

We will now describe in detail the steps involved in creating the TMLE estimate for the time to event data structure:

1. Set up the observed data in its long form as presented in Table 2.

2. Generate $\hat{Q}_{2}^{0}$, an estimate of the intensity process of the event of interest, $Q_{20}$. We suggest using a data adaptive technique such as Super Learner for binary outcomes to generate this estimate.

3. Estimate the treatment and censoring mechanisms, $\hat{g}_{1}$ and $\hat{g}_{2}$, also using data adaptive methods. Estimation of the censoring mechanism will also require that the data 
be represented in its long form; however, the outcome will now be censoring and the event of interest will act as the censoring event. Estimation of the treatment mechanism just requires that the data be set up the same way as for a standard logistic regression. These estimates will not be updated in the targeting steps below.

4. Evaluate $\hat{h}_{a}^{0}(t, A, W)$, the time dependent covariate based on the initial estimates, $\hat{Q}_{2}^{0}, \hat{g}_{1}$, and $\hat{g}_{2}$, for each line in the data set according to equation 10 above. Table 3 illustrates what the data set should look like after including $\hat{h}_{a}^{0}(t, A, W)$ for the four observations initially presented in Table 1 . As an example, let's consider the values of $\hat{h}_{a}^{0}(t, A, W)$ for the first six rows of Table 3 for estimating $\Psi_{1}\left(p_{0}\right)(2)$, or the probability of survival past time 2 , setting individuals to treatment level 1 . These are the rows in the long form data set for subject 1 . For the first row in the data set $\hat{h}_{1}(t=1, A=1, W=(1,1,439))$ will evaluate to:

$$
-\frac{1}{\hat{g}_{10}(A=1 \mid W=(1,1,439))} \frac{\hat{S}^{0}(2 \mid A=1, W=(1,1,439))}{\hat{S}^{0}(1 \mid A=1, W=(1,1,439))},
$$

and for the second row $\hat{h}_{1}(t=2, A=1, W=(1,1,439))$ will evaluate to:

$$
-\frac{1}{\hat{g}_{10}(A=1 \mid W=(1,1,439))(1-\hat{g}(1 \mid A=1, W=(1,1,439)))},
$$

and for all of the other rows $\hat{h}_{1}(t, A, W)$ will evaluate to zero since $I\left(t \leq t_{k}\right)$ will evaluate to zero for all time points past $t_{k}$. Alternatively, had we been interested in estimating $\Psi_{0}\left(p_{0}\right)(2)$ all of the rows for subject 1 would have had $\hat{h}_{0}(t, A, W)$ equal to zero since $I(A=a)$ would evaluate to zero.

5. Construct the first update $\hat{Q}_{2}^{1}$ of $\hat{Q}_{2}^{0}$. This is done by by specifying the following fluctuation of $\hat{Q}_{2}^{0}$ according to a parametric logistic regression model:

$$
\operatorname{logit}\left[\hat{Q}_{2}^{0}(\epsilon)\left(N_{1}(t), A, W\right)\right]=\operatorname{logit}\left[\hat{Q}_{2}^{0}\left(N_{1}(t), A, W\right)\right]+\epsilon h(t, A, W) \text {, }
$$

and obtaining $\hat{\epsilon}$ using standard parametric maximum likelihood estimation. $\hat{Q}_{2}^{1}=$ $\hat{Q}_{2}^{0}(\hat{\epsilon})$ is the first step targeted maximum likelihood estimate of $Q_{20}$.

6. Steps four and five are now iterated using the new estimate of $Q_{20}$ obtained in step 5 as the initial estimate until $\epsilon$ converges to zero. In each progression the k-th step targeted maximum likelihood estimate is produced $\hat{Q}_{2}^{k}$. Here, and in the discussions below we will occasionally express $\hat{Q}_{2}^{k-1}(\hat{\epsilon})$ as $\hat{Q}_{2}^{k}$, suppressing the $\epsilon$ for convenience 
despite the fact that all targeting steps are constructed based on estimating an additional fluctuation parameter. Once $\hat{\epsilon}$ is less than some pre-specified value $\delta$ (e.g., .001), the iterations are discontinued and the resulting estimator is $\hat{Q}_{2}^{*}$, the targeted maximum likelihood estimate of $Q_{20}$

7. Finally, construct $\Psi\left(\hat{Q}_{2}^{*}\right)$ by applying the parameter mapping $\Psi$, resulting in the targeted maximum likelihood estimate of $\Psi\left(Q_{0}\right)$.

Note, that had the data been generated according to the same causal graph as presented in Figure 1 but a different parameter was of interest, the exact same steps would be used to construct the TMLE estimate but with a different $h(t, A, W)$ specific to that parameter's efficient influence curve.

\begin{tabular}{ccccccccc}
\hline Subject & $t$ & $N_{1}(t)$ & $N_{2}(t)$ & A & Sex & MSM & Baseline CD4 & $\hat{h}_{a}(t, A, W)$ \\
\hline 1 & 1 & 0 & 0 & 1 & 1 & 1 & 439 & $\hat{h}(t=1, A=1, W=(1,1,439))$ \\
1 & 2 & 0 & 0 & 1 & 1 & 1 & 439 & $\hat{h}(t=1, A=1, W=(1,1,439))$ \\
1 & 3 & 0 & 0 & 1 & 1 & 1 & 439 & $\hat{h}(t=1, A=1, W=(1,1,439))$ \\
1 & 4 & 0 & 0 & 1 & 1 & 1 & 439 & $\hat{h}(t=1, A=1, W=(1,1,439))$ \\
1 & 5 & 0 & 0 & 1 & 1 & 1 & 439 & $\hat{h}(t=1, A=1, W=(1,1,439))$ \\
1 & 6 & 1 & 0 & 1 & 1 & 1 & 439 & $\hat{h}(t=1, A=1, W=(1,1,439))$ \\
2 & 1 & 0 & 0 & 1 & 1 & 0 & 71 & $\hat{h}(t=1, A=1, W=(1,0,71))$ \\
2 & 2 & 0 & 0 & 1 & 1 & 0 & 71 & $\hat{h}(t=2, A=1, W=(1,0,71))$ \\
2 & 3 & 0 & 0 & 1 & 1 & 0 & 71 & $\hat{h}(t=3, A=1, W=(1,0,71))$ \\
2 & 4 & 0 & 0 & 1 & 1 & 0 & 71 & $\hat{h}(t=4, A=1, W=(1,0,71))$ \\
2 & 5 & 0 & 1 & 1 & 1 & 0 & 71 & $\hat{h}(t=5, A=1, W=(1,0,71))$ \\
3 & 1 & 0 & 0 & 0 & 1 & 0 & 118 & $\hat{h}(t=1, A=0, W=(1,0,118))$ \\
3 & 2 & 0 & 0 & 0 & 1 & 0 & 118 & $\hat{h}(t=2, A=0, W=(1,0,118))$ \\
3 & 3 & 0 & 0 & 0 & 1 & 0 & 118 & $\hat{h}(t=3, A=0, W=(1,0,118))$ \\
3 & 4 & 0 & 0 & 0 & 1 & 0 & 118 & $\hat{h}(t=4, A=0, W=(1,0,118))$ \\
3 & 5 & 0 & 1 & 0 & 1 & 0 & 118 & $\hat{h}(t=5, A=0, W=(1,0,118))$ \\
4 & 1 & 0 & 0 & 1 & 0 & 0 & 106 & $\hat{h}(t=1, A=1, W=(0,0,106))$ \\
4 & 2 & 0 & 0 & 1 & 0 & 0 & 106 & $\hat{h}(t=2, A=1, W=(0,0,106))$ \\
4 & 3 & 1 & 0 & 1 & 0 & 0 & 106 & $\hat{h}(t=3, A=1, W=(0,0,106))$ \\
\hline
\end{tabular}

Table 3: Example of HIV analysis data of Section 12 in long form with additional clever covariate $\hat{h}(t, A, W)$. 


\section{Inference For Targeted Maximum Likelihood Estimation Of Treatment Specific Survival}

In this section we will address the construction of confidence intervals for TMLEs. The targeted maximum likelihood estimator, $\hat{Q}^{*}$, and corresponding estimate of $g_{0}, \hat{g}=\left(\hat{g}_{1}, \hat{g}_{2}\right)$ solves the efficient influence curve/score equation:

$$
0=\sum_{i=1}^{n} D^{*}\left(\hat{Q}^{*}, \hat{g}\right)\left(O_{i}\right),
$$

where, $D^{*}\left(Q_{0}, g_{0}\right)=D^{*}\left(Q_{0}, g_{0}, \Psi\left(Q_{0}\right)\right)$ is the efficient influence curve presented above in Equation 9 . One can also state that $\Psi\left(\hat{Q}^{*}\right)$ solves the estimating equation in $\psi_{0}$ :

$$
0=\sum_{i} D^{*}\left(\hat{Q}^{*}, \hat{g}, \Psi\left(\hat{Q}^{*}\right)\right)\left(O_{i}\right)
$$

defined by this efficient influence curve equation. Under regularity conditions, it can be shown that $\Psi\left(\hat{Q}^{*}\right)$ is asymptotically linear with an influence curve $D^{*}\left(Q, g_{0}, \psi_{0}\right)+D_{1}$ for the case that $\hat{Q}^{*}$ possibly converges to a mis-specified $Q$, and $\hat{g}$ converges to the true $g_{0}$ (van der Laan and Robins (2003), Section 2.3.7). In van der Laan, Gruber (2009) this asymptotic linearity result is generalized to hold for targeted MLE when $\hat{Q}$ converges to a possibly misspecified $Q$, and $\hat{g}$ converges to a true conditional censoring/treatment mechanism $g_{0}(Q)$ that conditions on all covariates the residual bias $Q-Q_{0}$ still depends on. The latter type of consistency of $\left(\hat{Q}^{*}, \hat{g}\right)$ we will refer to as the collaborative consistency of $\left(\hat{Q}^{*}, \hat{g}\right)$ for $\psi_{0}$. If $Q=Q_{0}$, the contribution $D_{1}$ equals zero. In addition, if $\hat{Q}^{*}$ converges to a misspecified $Q$, then in most situations the collaborative estimator will converge to the true $g_{0}$ conditioning on all variables $Q_{0}$ depends on, in which case it has been proven in Section 2.3.7 (van der Laan, Robins, 2003) that $D^{*}\left(Q, g_{0}, \psi_{0}\right)+D_{1}$ has smaller variance than the variance of $D^{*}\left(Q, g_{0}, \psi_{0}\right)$.

Based on these results, when an estimate $\Psi\left(\hat{Q}^{*}\right)$ solves the efficient influence curve equation, relying on the collaborative robustness of efficient influence curve and collaborative consistency of $\left(\hat{Q}^{*}, \hat{g}\right)$ for $\psi_{0}$, inference may be based on the empirical variance of the efficient influence curve $D^{*}$ itself at the limit of $\hat{Q}^{*}, \hat{g}$. Thus, the asymptotic variance of $n^{1 / 2}\left(\hat{\psi}_{a}^{*}-\Psi_{a}\right)$ may be estimated by:

$$
\hat{\sigma}^{2}=\frac{1}{n} \sum_{i=1}^{n} D_{a}^{* 2}\left(\hat{Q}^{*}, \hat{g}\right)\left(O_{i}\right) .
$$

Now 95 percent confidence intervals for the treatment specific survival curve at a particular time point may be constructed under the normal distributuion in the following way: 


$$
\hat{\psi}_{a}^{*} \pm 1.96 \frac{\hat{\sigma}}{\sqrt{n}}
$$

Alternatively, bootstrap 95 percent confidence intervals may be constructed. It will be shown in the simulation results below that, in certain situations, the bootstrap confidence intervals produce better coverage in finite samples.

The $\delta$-method may be used to obtain estimates of the influence curves of the parameters of interests expressed in Equations 4 through 6, as well as other parameters of interest which are a function of the treatment specific survival probabilities. The estimated influence curves for these parameters are:

$$
\begin{aligned}
D_{R D}^{*}\left(\hat{p}^{*}\right)\left(t_{k}\right) & =D_{1}^{*}\left(\hat{p}^{*}\right)\left(t_{k}\right)-D_{0}^{*}\left(\hat{p}^{*}\right)\left(t_{k}\right), \\
D_{R R}^{*}\left(\hat{p}^{*}\right)\left(t_{k}\right) & =-\frac{1}{1-\hat{\psi}_{1}^{*}\left(t_{k}\right)} D_{1}^{*}\left(\hat{p}^{*}\right)\left(t_{k}\right)+\frac{1}{1-\hat{\psi}_{0}^{*}\left(t_{k}\right)} D_{0}^{*}\left(\hat{p}^{*}\right)\left(t_{k}\right), \\
D_{R H}^{*}\left(\hat{p}^{*}\right)\left(t_{k}\right) & =-\frac{1}{\hat{\psi}_{1}^{*}\left(t_{k}\right) \log \left(\hat{\psi}_{1}^{*}\right)} D_{1}^{*}\left(\hat{p}^{*}\right)\left(t_{k}\right)+\frac{1}{\hat{\psi}_{0}^{*}\left(t_{k}\right) \log \left(\hat{\psi}_{0}^{*}\right)} D_{0}^{*}\left(\hat{p}^{*}\right)\left(t_{k}\right) .
\end{aligned}
$$

Confidence intervals may now be constructed for these parameters at a particular time point using the above estimates of the efficient influence curve. Furthermore, the estimated influence curve for estimates which are means of these parameters may be constructed by taking means of the estimated efficient influence curves (Equations 20 - 22) over the desired time points.

\section{Collaborative Targeted Maximum Likelihood Estimation Of Treatment Specific Survival}

Extending the TMLE estimate to a C-TMLE estimate requires generating a sequence of estimates of $g_{0}$ and corresponding sequence of TMLE estimates and then choosing from that sequence of TMLE estimates the one which has the minimum cross-validated risk. Thus, in order to implement the C-TMLE one must choose both a method for sequencing the estimates of $g_{0}$ and an appropriate loss function. In this section we will propose a way to sequence the estimates of $g_{0}$ as well as choose a loss function for the time to event setting.

Since the missingness mechanism, $g_{0}$, factorizes into both a treatment mechanism, $g_{10}$ and censoring mechanism, $g_{20}$, the sequence of estimates of $g_{0}$ must be a sequence of estimates of both the treatment and censoring mechanism. Thus, we propose a sequence of estimates where each step in the sequence either the censoring or treatment mechanism is more non-parametric than it was in the previous step. Since a priori dimension reductions 
of the covariate profiles can be employed, using main term regressions for these estimates is reasonable and lends itself nicely to defining a sequence of estimates of $g_{0}$.

Suppose one observes $A$, and $K$ baseline covariates $W_{1} \ldots W_{K}$ and $L$ is the last time point observed for any subject. First an initial estimate of $Q_{20}$ is constructed using a data adaptive method for the binary hazard regression such as Super Learner. This estimate is $\hat{Q}_{2}$ and is fit on all the data and held fixed. Next, define a sequence of $J$ moves $M_{0} \ldots M_{J}$, where $J=2 * K+1$. Each $M_{j}$ includes two estimates: a main term logistic regression estimate of $g_{10}$ and a main term logistic regression estimate of $g_{20} . M_{0}$ is composed of an estimate for $g_{10}$ using the logistic intercept model and for $g_{20}$ using a logistic regression non-parametrically fitting time:

$M_{0}=\left\{\begin{array}{l}g_{1 M_{0}}=\operatorname{logit}\left[P\left(A=1 \mid W_{1}, \ldots, W_{K}\right)\right]=\beta_{0} \\ g_{2 M_{0}}=\operatorname{logit}\left[P\left(N_{2}(t)=1 \mid A, W_{1}, \ldots, W_{K}\right)\right]=\alpha_{0}+\alpha_{1} I(t=2)+, \ldots,+\alpha_{L} I(t=L) .\end{array}\right.$

The next step in the sequence, $M_{1}$, consists of $g_{1 M_{1}}$ and $g_{2 M_{1}}$, which are constructed by adding a main term to either $g_{1 M_{0}}$ or $g_{2 M_{0}}$. So the set of possible $g_{1 M_{1}}$ is constructed by adding a main term from the set $\left\{W_{1}, \ldots, W_{K}\right\}$ to $g_{1 M_{0}}$ and the set of possible $g_{2 M_{1}}$ are constructed by adding a main term from the set $\left\{A, W_{1}, \ldots, W_{K}\right\}$ to $g_{2 M_{0}}$. The TMLE is evaluated at each possible $M_{1}$ and the main term that maximizes the increase in the penalized log-likelihood is the next move in the sequence. The estimate for which a main term is not added remains the same as in the previous step in the sequence. The variable which is added is then removed from the possible set of moves in the next step for that particular estimate. This process is continued until none of the possible steps increase the penalized log-likelihood. At that point an additional clever covariate $h_{2}$ is constructed, which is guaranteed to increase the penalized log-likelihood, and the TMLE estimate based off of $M_{j}$, where $j$ is the last completed step in the sequence, becomes the new initial $\hat{Q}_{2}$ for the TMLE algorithm. $M_{j+1}$ is now chosen based on adding a main term to $M_{j}$ as before. This process is continued until all $2 * K+1$ possible moves are completed adding new clever covariates and creating a new initial estimate of $Q_{20}$ when necessary. The number of moves completed indexes the sequence of candidate estimators of $g_{0}$ and this number of moves should be chosen with V-fold cross-validation.

An extension of the above sequencing procedure which uses data adaptive methods to estimate $g_{0}$ is also possible. One may incorporate more data adaptive techniques by allowing the main terms to be super learning fits of both the treatment and censoring mechanisms based on an increasing set of explanatory variables. Furthermore, the suggested sequencing algorithm presented here is one of many possible ways which the increasingly non-parametric estimates of $g_{0}$ may be constructed; in practice, alternative sequencing methods may be implemented.

In van der Laan and Gruber's initial paper on C-TMLE they discuss the importance of choosing a penalty to robustify the estimation procedure when the efficient influence curve blows up to infinity for certain models of $g_{0}$. The penalty term should make the criterion 
more targeted toward the parameter of interest while preserving the log-likelihood as the dominant term in situations where identifiability is not in jeopardy, as it is when there is no practical ETA violation. Thus, the penalty term should be asymptotically negligible but of importance in a sparse data setting. For this reason we initially chose to use the variance of the efficient influence curve as our penalty. The variance of the efficient influence curve is asymptotically negligible relative to the log-likelihood and in situations where there is an ETA violation, as in the case where $\hat{g}_{1}$ or $\prod_{i=1}^{t_{-}}\left[1-\hat{g}_{2}(i \mid A, W)\right]$ are very close to zero for a given subject, it will severely penalize the log-likelihood. However, the problem with this penalty for estimating the treatment specific survival curve is that it does not result in severe enough of a penalty in regions of $W$ where $a$ is not observed in the data. For example, if the curve is estimated when $\mathrm{A}=1$, many times one does not observe $A=1$ in regions of $W$ where it is unlikely to occur so the variance of the influence curve does not blow up as desired. For this reason we use an alternative version of the variance of the influence curve which first conditions on $A$ and then averages over all levels of $W$ observed in the data set:

$$
\frac{1}{n} \sum_{i}^{n} \sum_{t}^{t_{k}} \frac{1}{\hat{g}_{1}(A=a \mid W) \prod_{i=1}^{t_{-}}\left[1-\hat{g}_{2}(i \mid A, W)\right]} \frac{\hat{S}\left(t_{k} \mid A, W\right)}{\hat{S}(t \mid A, W)} I\left(t \leq t_{k}\right) \lambda(1-\lambda)
$$

where

$$
\lambda=\hat{Q}_{2}\left(N_{1}(t)=1, A=a, W\right) .
$$

The above penalty becomes large when the probability $A=a$ is small even for values of $W$ for which $A=a$ is not observed in the data. Thus, this penalty is sensitive to lack of identifiability, including theoretical non-identifiability.

Now that a sequence of models of $g_{0}$ and a penalized loss function have been defined, the C-TMLE algorithm may be implemented. Here is a summary of the steps involved:

1. Generate $\hat{Q}_{2}$, an estimate of $Q_{20}$ using a data adaptive technique such as Super Learner for binary outcomes.

2. Use cross-validation with the log-likelihood loss function penalized by equation 23 to choose the number of moves using the sequencing algorithm presented above.

3. Implement the sequencing algorithm on the full data set for the chosen number of moves.

4. The resulting $\hat{Q}_{2}^{*}$ from the TMLE indexed by the chosen number of moves is the C-TMLE estimate of of the hazard.

5. Construct the substitution estimator $\Psi\left(\hat{Q}_{2}^{*}\right)$ which is the C-TMLE estimate of the parameter of interest. 
Several variations on the above proposed sequencing algorithm and penalized likelihood were also explored to see if they would produce more robust estimates. The variations included:

1. Trimming - The observations which led to identifiability problems were removed from fitting $g$. This was done in order to get estimates which were not as influenced by outlying values in $W$ that were highly predictive of treatment/censoring.

2. Truncation - The observations which led to identifiability problems were set to a minimum probability. All subjects who had a treatment mechanism which predicted treatment less than $\mathrm{X}$ percent of the time, where $\mathrm{X}$ is a low probability were set to $\mathrm{X}$ percent. X could be say 5 or 10 percent.

3. Using Binary Covariates - Transforming the continuous variables to binary variables which are indicators of the quantiles of the original variable. Therefore, if one chose to do quartiles three indicator variables would be created for the top three quartiles which would take a 1 or a zero depending on what quartile an individual was in. When all three variables are zero the individual would be in the lowest quartile. This allows the C-TMLE algorithm to only adjust for the regions which do not cause ETA violations as opposed to the entire variable. Thus, the larger the number of binary variables constructed from an initial covariate, the more flexibility the CTMLE algorithm has. However, too many binary variables for a single continuous covariate may contribute to loss of signal and a large increase in the computation time of the algorithm.

4. Using Mean Square Error instead of log-likelihood as a loss function.

Using binary covariates had the largest positive effect on producing robust estimates and trimming and truncation had little effect on the results in the simulations presented below. After many simulations it was revealed that the log-likelihood does a much better job of distinguishing between models than the mean square error loss function, at least for the time to event outcomes analyzed here. Furthermore, a dimension reduction step in between the initial fit of $Q_{20}$ and the sequencing step improved computation time tremendously. This was done by removing all variables from the sequencing step that fell below a certain cutoff in terms of association with the outcome of interest after accounting for the initial fit. Univariate regression was performed with the initial estimate as an offset and all variables which fell below a 10 percent false discovery rate (FDR) adjusted p-value were no longer considered in the sequencing step. All C-TMLE estimates presented in the remainder of this paper include the dimension reduction step and use binary covariates for the secondary sequencing step. 


\section{Inference For Collaborative Targeted Maximum Likeli- hood Estimation Of Treatment Specific Survival}

We refer to section 9. It is shown in van der Laan and Gruber 2010 that Collaborative Targeted Maximum Likelihood estimators and corresponding estimates $\hat{g}$ solve the efficient influence curve equation. Thus, confidence intervals may be constructed for C-TMLE the same way they are constructed for TMLE in section 9.

\section{Simulation Study}

In the following sections we present the results of a simulation study which illustrates the advantages of the C-TMLE compared to alternative methods. The simulation consists of data sets generated under three scenarios: no ETA violation, medium ETA violation, and high ETA violation. Within each scenario estimates are presented for each of the methods using the true model for $\mathrm{Q}$, as if it was known a priori, and a purposely mis-specified model for Q. The methods are then evaluated based on how they performed in terms of bias, variance, and their ability to perform inference.

The simulated data were generated in the following way for each subject in the data set:

1. The baseline covariates $W=\{W 1, W 2, W 3, W 4, W 5\}$ were generated from a multivariate normal with mean $=0$, variance $=1$, and covariance $=.2$. If any $\mathrm{W}$ were greater than 2 or less than -2 they were set to 2 or -2 , respectively, to ensure that the treatment and censoring mechanisms were appropriately bounded.

2. The treatment $\mathrm{A}$ was generated as a binomial in the following way:

$P(A=1 \mid W)=\operatorname{logit}^{-1}(.4+.4 \times W 1+.4 \times W 2-.5 \times W 3+\log ($ ETA OR $) \times W 4)$

where the ETA OR is the ETA odds ratio and $W 4$ is the baseline covariate responsible for violations in the ETA assumption in the simulated data sets. ETA OR equals 1 for the scenario under no ETA violation, 10 for medium ETA violation, and 15 for the high ETA violation scenario.

3. The event process was generated using the following hazard at each point in time, $\mathrm{t}$ :

$$
P\left(N_{1}(t)=1 \mid A, W\right)=\operatorname{logit}^{-1}(.25-.6 \times W 1-.6 \times W 2-.6 \times W 3-1 \times A)
$$

4. Similarly, the censoring process was generated using the following hazard at each point in time, $\mathrm{t}$ :

$$
P\left(N_{2}(t)=1 \mid A, W\right)=\operatorname{logit}^{-1}(-3-.1 \times W 1-.1 \times W 2-.1 \times W 3-.1 \times A)
$$


Under each level of ETA violation 500 data sets of 500 observations were generated according to the above process. Once the data sets were generated each of the following methods were used to estimate the parameter of interest, which is the treatment specific survival curve for $\mathrm{A}=1$ at time 2 :

1. IPW estimate, $\hat{\psi}_{1}^{I P W}(2)$. Where $g_{1}$ and $g_{2}$ were estimated based off of the known model.

2. G-comp estimate, $\hat{\psi}_{1}^{g-c o m p}(2)$.

3. AIPW estimate, $\hat{\psi}_{1}^{A I P W}(2)$. Where $g_{1}$ and $g_{2}$ were estimated based off of the known model.

4. TMLE estimate, $\hat{\psi}_{1}^{T M L E}(2)$. Where $g_{1}$ and $g_{2}$ were estimated based off of the known model.

5. AIPW w/o ETA Variable estimate. Where $g_{1}$ and $g_{2}$ were estimated based off of the known model. However, $W 4$, the variable responsible for violations in the ETA assumption, was not adjusted for in estimating the treatment mechanism, $g_{1}$.

6. TMLE w/o ETA Variable estimate. Where $g_{1}$ and $g_{2}$ were estimated based off of the known model. However, $W 4$, the variable responsible for violations in the ETA assumption, was not adjusted for in estimating the treatment mechanism, $g_{1}$.

7. C-TMLE estimate, $\hat{\psi}_{1}^{C-T M L E}(2)$, using the method described in the section 10 with dimension reduction, a loss function penalized by the integrated version of the variance of the influence curve, and binary baseline covariates, $W$, split at the $33 \mathrm{rd}$ and 66 th precentile.

Each of the above methods were implemented twice for each data set; once using the known model for $Q$ and once using a purposely mis-specified model for $Q$ which only included $A$ and $W 5$ as main terms in the logistic hazard regression to obtain $\hat{Q}$. Note, that $W 5$ is just a noise variable and does not play a role in the outcome process, censoring process, or treatment mechanism. Estimates 5 and 6 listed above are not estimates one could implement based on a real data set but were evaluated to compare the C-TMLE algorithm to methods which a priori knew and removed a variable which is causing identifiability problems. Furthermore, all of the estimation methods except for C-TMLE were given the true model for $g$, an advantage they would not have when analyzing real data. In a real data analysis model selection which uses a loss function for prediction of the treatment and censoring mechanism would be implemented.

Up until now, the simulation study has been concerned with a variable that causes violations in ETA and is not a cause of the outcome of interest. However, many times the problem variable may be a confounder, and thus has a causal effect on both the treatment, 
$A$, and the outcome of interest. In order to evaluate the methods in such a scenario, we reran the high ETA violation scenario with a minor change. The treatment A was generated as a binomial in the following way:

$$
P(A=1 \mid W)=\operatorname{logit}^{-1}(.4+\log (15) \times W 1+.4 \times W 2-.5 \times W 3)
$$

Therefore, instead of varying the odds ratio for $W 4$ we set the odds ratio for $W 1$, one of the variables that effects the hazard of the event of interest, to 15.

In the following two sections we will present the results of the simulation study. In Section 13 we will compare the methods in terms of bias and mean square error and in Section 14 in terms of inference.

\section{Simulation Study Results: Estimates}

Tables 4 through 6 display the simulation results for the scenarios where the variable causing identifiability concerns is not a confounder. The true value of the parameter being estimated is .462. It can be seen in Table 4 that all of the methods produce unbiased estimates of the parameter of interest when the initial model for $\mathrm{Q}$ is specified correctly. However, when the initial model for Q is mis-specified, the G-comp estimate is biased. The IPW estimate is the same for the mis-specified and correctly specified Q since this estimate does not depend on an estimate of $\mathrm{Q}$.

\begin{tabular}{lcccccc}
\hline & \multicolumn{2}{c}{ No ETA } & \multicolumn{2}{c}{ Medium ETA } & \multicolumn{2}{c}{ High ETA } \\
\multicolumn{1}{c}{ Method } & Known Q & Bad Q & Known Q & Bad Q & Known Q & Bad Q \\
\hline TMLE & 0.460 & 0.460 & 0.463 & 0.460 & 0.465 & 0.458 \\
G-comp & 0.461 & 0.473 & 0.461 & 0.499 & 0.461 & 0.501 \\
IPW & 0.460 & 0.460 & 0.461 & 0.461 & 0.460 & 0.460 \\
AIPW & 0.460 & 0.460 & 0.463 & 0.464 & 0.464 & 0.466 \\
AIPW w/o ETA variable & 0.460 & 0.460 & 0.460 & 0.455 & 0.460 & 0.455 \\
TMLE w/o ETA variable & 0.460 & 0.460 & 0.460 & 0.455 & 0.460 & 0.455 \\
C-TMLE & 0.460 & 0.463 & 0.460 & 0.460 & 0.461 & 0.463 \\
\hline
\end{tabular}

Table 4: Simulation Results: Mean Of 500 Estimates (True Value Of Parameter Is .462). Variable Causing Violation In ETA Is Not A Confounder.

The fact that all of the methods produce unbiased estimates of the parameter of interest, even in the moderate sample sizes examined here, suggests that bias should not be the standard by which these methods are judged. Assessing the methods in terms of mean square error (MSE) begins to distinguish the methods from one another. Table 5 presents the root mean square error, relative efficiency (on the variance scale), and the efficiency 
bound for each scenario. Again, in the no ETA scenario MSE does not distinguish the methods from one another as they all have essentially the same MSE. However, as the ETA becomes larger some of the methods begin to showcase their advantages while others lose all stability. The following observations may be made based off of Table 5:

\begin{tabular}{lcccccc}
\hline & \multicolumn{2}{c}{ No ETA } & \multicolumn{2}{c}{ Medium ETA } & \multicolumn{2}{c}{ High ETA } \\
\multicolumn{1}{c}{ Method } & Known Q & Bad Q & Known Q & Bad Q & Known Q & Bad Q \\
\hline Efficiency Bound & \multicolumn{2}{c}{0.028} & \multicolumn{2}{c}{0.046} & & \\
& & & & & \\
Root Mean Square Error & & & & & \\
TMLE & 0.029 & 0.029 & 0.051 & 0.056 & 0.062 & 0.065 \\
G-comp & 0.027 & 0.030 & 0.028 & 0.047 & 0.028 & 0.049 \\
IPW & 0.029 & 0.029 & 0.071 & 0.071 & 0.106 & 0.106 \\
AIPW & 0.029 & 0.029 & 0.054 & 0.061 & 0.070 & 0.081 \\
AIPW w/o ETA variable & 0.029 & 0.029 & 0.030 & 0.031 & 0.029 & 0.031 \\
TMLE w/o ETA variable & 0.029 & 0.029 & 0.030 & 0.031 & 0.029 & 0.030 \\
C-TMLE & 0.028 & 0.031 & 0.029 & 0.037 & 0.029 & 0.040 \\
& & & & & & \\
Relative Efficiency & & & & & & \\
TMLE & 1.0 & 1.1 & 1.2 & 1.5 & 1.0 & 1.2 \\
G-comp & 0.9 & 1.1 & 0.4 & 1.0 & 0.2 & 0.7 \\
IPW & 1.0 & 1.0 & 2.4 & 2.4 & 3.1 & 3.1 \\
AIPW & 1.0 & 1.1 & 1.4 & 1.8 & 1.3 & 1.8 \\
AIPW w/o ETA variable & 1.0 & 1.1 & 0.4 & 0.5 & 0.2 & 0.3 \\
TMLE w/o ETA variable & 1.0 & 1.1 & 0.4 & 0.4 & 0.2 & 0.3 \\
C-TMLE & 1.0 & 1.2 & 0.4 & 0.7 & 0.2 & 0.4 \\
\hline
\end{tabular}

Table 5: Simulation Results: Root Mean Square Error And Relative Efficiency. Variable Causing Violation In ETA Is Not A Confounder.

1. The IPW estimate is highly unstable with increasing ETA. In fact, the C-TMLE is six times more efficient when $Q$ is estimated well and almost 3.5 times more efficient when $Q$ is mis-specified in the medium ETA scenario. For the High ETA case, the C-TMLE is 15.5 times more efficient for a well estimated $Q$ and eight times more efficient when $Q$ is mis-specified.

2. The AIPW estimates also begin to lose stability with increasing ETA but not as much as the IPW estimates. The C-TMLE is 3.5 more times efficient than the AIPW estimate for a correctly specified $Q$ and 2.6 times more efficient for a mis-specified $Q$ in the medium ETA scenario. For the high ETA scenario, the C-TMLE is 6.5 and 


\begin{tabular}{|c|c|c|c|c|c|c|}
\hline \multirow[b]{2}{*}{ Method } & \multicolumn{2}{|c|}{ No ETA } & \multicolumn{2}{|c|}{ Medium ETA } & \multicolumn{2}{|c|}{ High ETA } \\
\hline & Known $\mathrm{Q}$ & Bad Q & Known Q & Bad Q & Known Q & Bad Q \\
\hline Mean Number of Moves & 0.1 & 13.4 & 0.1 & 10.5 & 0.1 & 10.1 \\
\hline \multicolumn{7}{|c|}{ Percent of Time C-TMLE Algorithm Chose: } \\
\hline Zero Moves & 0.98 & 0.00 & 0.98 & 0.06 & 0.99 & 0.08 \\
\hline W1 & 0.00 & 0.99 & 0.00 & 0.93 & 0.00 & 0.89 \\
\hline W2 & 0.00 & 0.99 & 0.00 & 0.91 & 0.00 & 0.89 \\
\hline W3 & 0.00 & 0.99 & 0.00 & 0.81 & 0.00 & 0.81 \\
\hline W4 (ETA Variable) & 0.01 & 0.84 & 0.00 & 0.47 & 0.00 & 0.41 \\
\hline W5 & 0.01 & 0.00 & 0.01 & 0.00 & 0.01 & 0.00 \\
\hline
\end{tabular}

Table 6: Simulation Results: Characteristics Of C-TMLE Algorithm. Variable Causing Violation In ETA Is Not A Confounder.

4.5 times more efficient for the respective ways of estimating $Q$.

3. The TMLE estimate, regardless of ETA, scenario tends to have a MSE which approaches the efficiency bound unlike the IPW or AIPW estimates.

4. The C-TMLE estimate is super efficient and even as the ETA violation increases, the MSE of this estimate remains close to the level it was under no ETA violation. This is true wether $Q$ is fit well or mis-specified. The MSE is lower when $Q$ is specified correctly; however, it still out-performs any of the other estimates in terms of efficiency when $Q$ is mis-specified.

Figure 2 presents two scatter plots comparing the AIPW estimates to TMLE and CTMLE estimates in the high ETA scenario with a mis-specified Q. This is the scenario for which all estimates have the greatest difficulty in producing quality estimates (i.e. the scenario with the highest mean square error for all methods). The horizontal and vertical solid lines are the true value of the parameter being estimated, or 46.2 percent. Thus, points that are closer to the vertical black line than the horizontal black line are simulated data sets where TMLE or C-TMLE produced estimates closer to the truth than AIPW, and vice versa. These plots graphically depict the large difference in mean square error between, AIPW and TMLE, and, AIPW and C-TMLE, as seen in the last column of Table 5. In fact, the AIPW estimates range from 17.5 to 103 percent, the TMLE estimates range from 30.3 to 63.8 percent, and the C-TMLE estimates range from 35.6 to 59.2 percent. Thus, it becomes immediately clear that the AIPW does not respect the global restraints of the model by producing an estimate which is not a probability (One of the estimates is greater than 100 percent). In addition, the AIPW estimate is an empirical mean of an unbounded function of the data (Equation 11) and thus when the estimates of $g_{0}$ are close 
to zero the contribution from one observation may be too large or even infinite. Whereas, the TMLE and C-TMLE estimates are empirical means of probabilities (Equation 12). By virtue of being substitution estimators each observations contribution is bounded and may be no larger than 1 . The advantage of being substitution estimator is directly observed in the simulation results by the smaller mean squared error for TMLE than AIPW.

Table 6 presents some characteristics of the C-TMLE algorithm including how many, and which moves were chosen under each scenario. When $Q$ is specified well the C-TMLE algorithm makes very few moves and, in almost all cases, it makes zero moves leaving the intercept models for $g$. However, when $Q$ is mis-specified the C-TMLE algorithm chooses more moves and attempts to adjust in $g$ for the variables that were not adjusted for in the initial estimate of $Q$. Also, the algorithm resists choosing the region of the variable which causes the ETA violations illustrated by the fact that $W 4$ is selected less and less as ETA increases.

Tables 7-9 display the results of the high ETA scenario, where $W 1$, a confounder, is the variable causing identifiability problems. The "No ETA" columns are the same as before. Table 7 displays the mean estimates for this scenario. As before when $Q$ is specified well, all of the estimates are unbiased. However, when $Q$ is mis-specified while the TMLE, IPW, and AIPW remain unbiased, the G-comp estimate is highly biased and the C-TMLE is slightly biased. The bias in the C-TMLE is due to the fact that it is not fully adjusting for $W 1$ when regions of that variable contribute to non-identifiability of the parameter of interest. This bias is compensated for by the fact that the C-TMLE does as well as any of the other methods in terms of mean square error. Furthermore, the AIPW estimate does twice as bad as the C-TMLE in terms of mean square error as seen in Table 8. Though the IPW estimate is behaving reasonably in terms of bias and MSE in this simulation, its potential to provide highly unstable estimates was displayed above.

\begin{tabular}{lcccc}
\hline \hline \multirow{2}{*}{ Method } & \multicolumn{2}{c}{ No ETA } & \multicolumn{2}{c}{ High ETA } \\
& Known Q & Bad Q & Known Q & Bad Q \\
\hline TMLE & 0.460 & 0.460 & 0.468 & 0.459 \\
G-comp & 0.461 & 0.473 & 0.462 & 0.552 \\
IPW & 0.460 & 0.460 & 0.461 & 0.461 \\
AIPW & 0.460 & 0.460 & 0.462 & 0.466 \\
AIPW w/o ETA variable & 0.460 & 0.460 & 0.462 & 0.533 \\
TMLE w/o ETA variable & 0.460 & 0.460 & 0.462 & 0.533 \\
C-TMLE & 0.460 & 0.463 & 0.462 & 0.482 \\
\hline
\end{tabular}

Table 7: Simulation Results: Mean Of 500 Estimates (True Value Of Parameter Is .462). Variable Causing Violation In ETA Is A Confounder.

Table 9 shows the characteristics of the C-TMLE algorithm. Again, when $Q$ is specified 


\begin{tabular}{lcccc}
\hline & \multicolumn{2}{c}{ No ETA } & \multicolumn{2}{c}{ High ETA } \\
\multicolumn{1}{c}{ Method } & \multicolumn{2}{c}{0.028} & \multicolumn{2}{c}{0.054} \\
& \multicolumn{5}{c}{ Known Q } & Bad Q & Known Q & Bad Q \\
\hline Efficiency Bound & & & & \\
Root Mean Square Error & 0.029 & 0.029 & 0.053 & 0.050 \\
TMLE & 0.027 & 0.030 & 0.029 & 0.094 \\
G-comp & 0.029 & 0.029 & 0.058 & 0.058 \\
IPW & 0.029 & 0.029 & 0.052 & 0.077 \\
AIPW & 0.029 & 0.029 & 0.032 & 0.076 \\
AIPW w/o ETA variable & 0.029 & 0.029 & 0.031 & 0.076 \\
TMLE w/o ETA variable & 0.028 & 0.031 & 0.031 & 0.055 \\
C-TMLE & & & & \\
& & & & \\
Relative Efficiency & 1.0 & 1.1 & 1.0 & 0.9 \\
TMLE & 0.9 & 1.1 & 0.3 & 3.0 \\
G-comp & 1.0 & 1.0 & 1.2 & 1.2 \\
IPW & 1.0 & 1.1 & 0.9 & 2.0 \\
AIPW & 1.0 & 1.1 & 0.3 & 2.0 \\
AIPW w/o ETA variable & 1.0 & 1.1 & 0.3 & 2.0 \\
TMLE w/o ETA variable & 1.0 & 1.2 & 0.3 & 1.1 \\
C-TMLE & & & &
\end{tabular}

Table 8: Simulation Results: Mean Squared Error. Variable Causing Violation In ETA Is A Confounder.

correctly very few moves are made and when it is mis-specified the algorithm adjusts by choosing a fuller model for $g$. As expected, that the C-TMLE algorithm is having a harder time choosing what variables to adjust for now that the ETA variable is a confounder. This can be seen by the fact that the algorithm continues to adjust for $W 1$ more often then it did for $W 4$ in table 6 . The algorithm uses the penalized loss function to weigh whether it is better to adjust for a variable which is associated with the outcome or remove it since it causes identifiability problems. In this case, the algorithm has chosen to adjust for at least some region of the variable a large percentage of the time. Had the algorithm decided to remove the variable completely from the adjustments, the estimates would be even more biased, and the MSE would be very large like the values seen for the TMLE w/o ETA variable estimate. This difference in MSE illustrates the value of generating binary variables and then running the C-TMLE algorithm on those as opposed to on the entire variable. 


\begin{tabular}{lcccc}
\hline \hline & \multicolumn{2}{c}{ No ETA } & \multicolumn{2}{c}{ High ETA } \\
\multicolumn{1}{c}{ Method } & Known Q & Bad Q & Known Q & Bad Q \\
\hline Mean Number of Moves & 0.103 & 13.389 & 0.134 & 8.448 \\
\multicolumn{4}{l}{ Percent of Time C-TMLE Algorithm Chose: } \\
Zero Moves & 0.98 & 0.00 & 0.97 & 0.12 \\
W1 & 0.00 & 0.99 & 0.00 & 0.88 \\
W2 & 0.00 & 0.99 & 0.00 & 0.80 \\
W3 & 0.00 & 0.99 & 0.00 & 0.59 \\
W4 & 0.01 & 0.84 & 0.01 & 0.48 \\
W5 & 0.01 & 0.00 & 0.02 & 0.00 \\
\hline
\end{tabular}

Table 9: Simulation Results: Characteristics of C-TMLE Algorithm

\section{Simulation Study Results: Inference. Variable Causing Violation In ETA Is A Confounder.}

We will now examine how the different estimators compare in producing valid 95 percent confidence intervals. Table 10 presents the percent of time the 95 percent influence curve based confidence intervals includes the true value, for the scenario where the ETA variable is not a confounder. Ideally, a well behaved method would produce confidence intervals that include the truth 95 percent of the time. Keep in mind that each scenario was only simulated 500 times so some variation from 95 percent is not unexpected. The confidence intervals for the G-comp method are not reported since there is no theory that suggests an appropriate way to construct such intervals. The AIPW is the only method which retains true 95 percent confidence intervals over all scenarios (Excluding the methods which don't adjust for the ETA variable since they are not feasible in a real data setting). The influence curve based confidence intervals of the TMLE begin to deteriorate with increasing ETA. The C-TMLE coverage probability also decreases with increasing ETA but not as quickly as for the TMLE. Table 11 shows the 95 percent coverage probabilities for the scenario that the ETA variable is a confounder. Again, the TMLE and C-TMLE coverage probabilities are less than 95 percent. Thus, we can conclude from Tables 10 and 11 that the theoretically valid asymptotic, influence curve based, confidence intervals are not producing proper coverage in the finite samples when identifiability is an issue. Furthermore, as the parameter becomes more non-identifiable the coverage probabilities further deteriorate.

Tables 10 and 11 also present the mean width of the influence curve based confidence intervals for each estimator. These tables show that while the AIPW's confidence intervals have proper coverage the width of these intervals are larger than the intervals of both the TMLE and C-TMLE estimation methods. In fact, the average AIPW interval width for the 
"high ETA" scenario with mis-specified initial Q is almost twice as large as the C-TMLE interval width in Table 10. In the scenario where the ETA variable is a confounder, under "high ETA" and mis-specified Q, the AIPW intervals are on average 67 percent larger than the C-TMLE intervals.

The scatter plots in Figures 2 through 4 are intended to compare the performance of influence curve based confidence intervals between AIPW and TMLE or C-TMLE. All three Figures are for the high ETA scenario with a mis-specified Q, the scenario which had the lowest coverage probabilities for TMLE. Figure 2 presents a scatterplot of the AIPW estimates versus TMLE and C-TMLE and was initially discussed in the previous section.

We will now reexamine these plots considering their implications on inference. The plots distinguish four different situations with regard to the confidence intervals. A blue circle indicates that for a particular data set the influence curve based confidence intervals include the truth for both the AIPW estimate and the TMLE/C-TMLE estimate. The TMLE/C-TMLE confidence bars could be thought of as going from left to right and the confidence bars for the AIPW would go up and down. Thus a blue circle would indicate that the confidence interval for the AIPW estimate would extend up and down beyond the horizontal line (indicating the true value on the AIPW axis) and the confidence interval for the TMLE/C-TMLE would extend left to right beyond the vertical solid line (indicating the true value on the TMLE/C-TMLE axis). Similarly, a green triangle would have a confidence interval that includes the horizontal line (The AIPW interval includes the truth) but the confidence interval would not include the vertical solid line (The TMLE or C-TMLE interval does not include the truth). The red plus indicates that the TMLE/C-TMLE interval includes the truth and the AIPW does not and the light blue $\mathrm{x}$ indicates that neither estimates confidence interval includes the truth.

The first major conclusion to draw from these plots is that even though the AIPW intervals include the truth the correct percentage of the time in many cases the intervals are extremely large rendering these estimates useless. This is illustrated by the fact that there are AIPW estimates whose intervals include the truth (blue circles and green triangles) far from the horizontal line. Second, the data sets for which AIPW estimates have large intervals are not the ones for which the TMLE/C-TMLE are having difficulty with inference. In fact, the TMLE/C-TMLE influence curve based confidence intervals include the truth for almost all data sets where the AIPW estimate is below .35 or above .60. The TMLE/CTMLE intervals which do not include the truth appear to just barely not include the truth as is illustrated by the fact that the green triangles and blue circles have a fairly well defined border on the x-axis. This suggests that a small adjustment to the TMLE/C-TMLE influence curve based confidence intervals would cause the TMLE/C-TMLE intervals to include the truth.

The scatter plots in Figure 3 depict the standard error for each of the 500 simulated data sets for both the AIPW estimates and TMLE estimates. Again different points are used to indicate which of the confidence intervals include the truth. The only difference between the top and bottom scatter plot is the range of the axis; thus, the bottom scatterplot focuses on 
the standard errors in a range where the confidence intervals are more useful. For standard errors larger than .15, the 95 percent confidence interval is approximately 6 in length, bordering on too large to be useful when estimating a probability which ranges between 0 and 1. Figure 4 shows these same plots for the AIPW and C-TMLE estimates. The top plot in Figure 3 shows that the AIPW confidence intervals tend to be larger than the TMLE confidence intervals in the region of standard errors which produce useful confidence intervals; however, in the region where the confidence intervals are not useful, the reverse is true. The average length of the AIPW confidence interval is .255 compared to .237 for the TMLE and .132 for the C-TMLE. Even though the AIPW estimates produce confidence intervals with proper coverage; they are larger than the TMLE intervals and almost twice as large as the C-TMLE intervals which have only slightly less coverage. It is also seen in Figure 4 that the standard errors are almost alway larger for the AIPW method than C-TMLE for this simulation. In fact, none of the standard errors for C-TMLE exceed .1 while 5.4 percent of the AIPW standard errors exceed .15. Thus, the difference in coverage probabilities (.94 vs. .89) is compensated for by the large percent of confidence intervals which, though they contain the truth, are too large to be practically useful.

As in Figure 2, Figures 3 and 4 show that data sets where the TMLE and C-TMLE intervals do not cover the true values and the AIPW intervals do correspond with data sets which are not outliers in terms of the estimates and standard errors produced by using AIPW methods (These correspond with green triangles in the plot). Thus, the TMLE and C-TMLE confidence intervals would include the truth if the intervals were slightly shifted to the left or the right. We hypothesize that this departure from normality is the result of a distribution of estimates which is slightly skewed in finite samples. This suggests that bootstrap methods, which use the .025 and .975 bootstrap quantiles to construct confidence intervals, would produce valid 95 percent confidence intervals. In order to test this hypothesis 500 additional data sets were generated according to the original simulation's High ETA scenario and bootstrap confidence intervals, both based on quantiles and estimated standard error, as well as influence curve based confidence intervals were compared for the TMLE. Note, this was not done for C-TMLE because of the prohibitive amount of time it would take to do for 500 data sets; however, for one data set it is a feasible method for inference and the bootstrap results for the TMLE intervals should hold for C-TMLE. These results are presented in Table 12. For the 500 newly simulated data sets the resulting coverage probability was 94 percent using quantile based bootstrap intervals, compared to 88 percent for bootstrap intervals based on the estimated standard error, and 87 percent using influence curve based confidence intervals. Furthermore, the average length of the bootstrap confidence intervals was $.21, .22$ and .26 respectively. This suggests that the quantile based bootstrap, which naturally accounts for the skewness in finite samples, is able to produce valid 95 percent confidence intervals. The lack of coverage of the standard error based bootstrap confidence intervals confirms that the skewness of the distribution of the estimates in finite samples is contributing to the poor influence curve based confidence intervals. This is because both of these methods depend on using the standard normal 
quantiles to generate confidence intervals. Not only do the quantile based bootstrap confidence intervals produce the proper coverage, but they also are 20 percent smaller than the influence curve based intervals. Thus, the quantile based bootstrap intervals should be the preferred method for constructing TMLE and C-TMLE confidence intervals.

\begin{tabular}{|c|c|c|c|c|c|c|}
\hline \multirow[b]{2}{*}{ Method } & \multicolumn{2}{|c|}{ No ETA } & \multicolumn{2}{|c|}{ Medium ETA } & \multicolumn{2}{|c|}{ High ETA } \\
\hline & Known Q & Bad Q & Known Q & Bad Q & Known Q & Bad Q \\
\hline \multicolumn{7}{|l|}{ Coverage Probabilities } \\
\hline TMLE & 0.94 & 0.96 & 0.86 & 0.85 & 0.80 & 0.83 \\
\hline IPW & 0.98 & 0.98 & 0.92 & 0.92 & 0.88 & 0.88 \\
\hline AIPW & 0.94 & 0.95 & 0.95 & 0.95 & 0.94 & 0.94 \\
\hline AIPW w/o ETA variable & 0.94 & 0.95 & 0.95 & 0.96 & 0.95 & 0.96 \\
\hline TMLE w/o ETA variable & 0.94 & 0.96 & 0.96 & 0.95 & 0.95 & 0.96 \\
\hline C-TMLE & 0.94 & 0.94 & 0.95 & 0.92 & 0.94 & 0.89 \\
\hline \multicolumn{7}{|c|}{ Mean Width of Confidence Intervals } \\
\hline TMLE & 0.11 & 0.12 & 0.19 & 0.21 & 0.21 & 0.24 \\
\hline IPCW/IPTW & 0.14 & 0.14 & 0.24 & 0.24 & 0.27 & 0.27 \\
\hline AIPW & 0.11 & 0.12 & 0.18 & 0.22 & 0.20 & 0.25 \\
\hline AIPW w/o ETA variable & 0.11 & 0.12 & 0.11 & 0.12 & 0.11 & 0.12 \\
\hline TMLE w/o ETA variable & 0.11 & 0.12 & 0.11 & 0.12 & 0.11 & 0.12 \\
\hline C-TMLE & 0.11 & 0.12 & 0.11 & 0.13 & 0.11 & 0.13 \\
\hline
\end{tabular}

Table 10: Simulation Results: 95 Percent Wald-Type Confidence Interval Coverage Probabilities And Mean Width Of Confidence Intervals. Variable Causing Violation In ETA Is Not A Confounder.

\section{Discussion}

Ultimately, a choice must be made to implement an estimation method which behaves the best across the largest number of possible scenarios. The simulations presented here illustrate the advantages of the C-TMLE methodology for estimating causal parameters when analyzing time to event outcomes. The results show that the C-TMLE method does at least as well as the best estimate under every scenario and, in many of the more realistic scenarios, behaves much better than the next best estimate in terms of both bias and variance. Unlike other estimators which rely on external estimates of nuisance parameters, the C-TMLE algorithm estimates these parameters with consideration for the parameter of interest. The C-TMLE is an entirely a priori specified method which accounts for the 

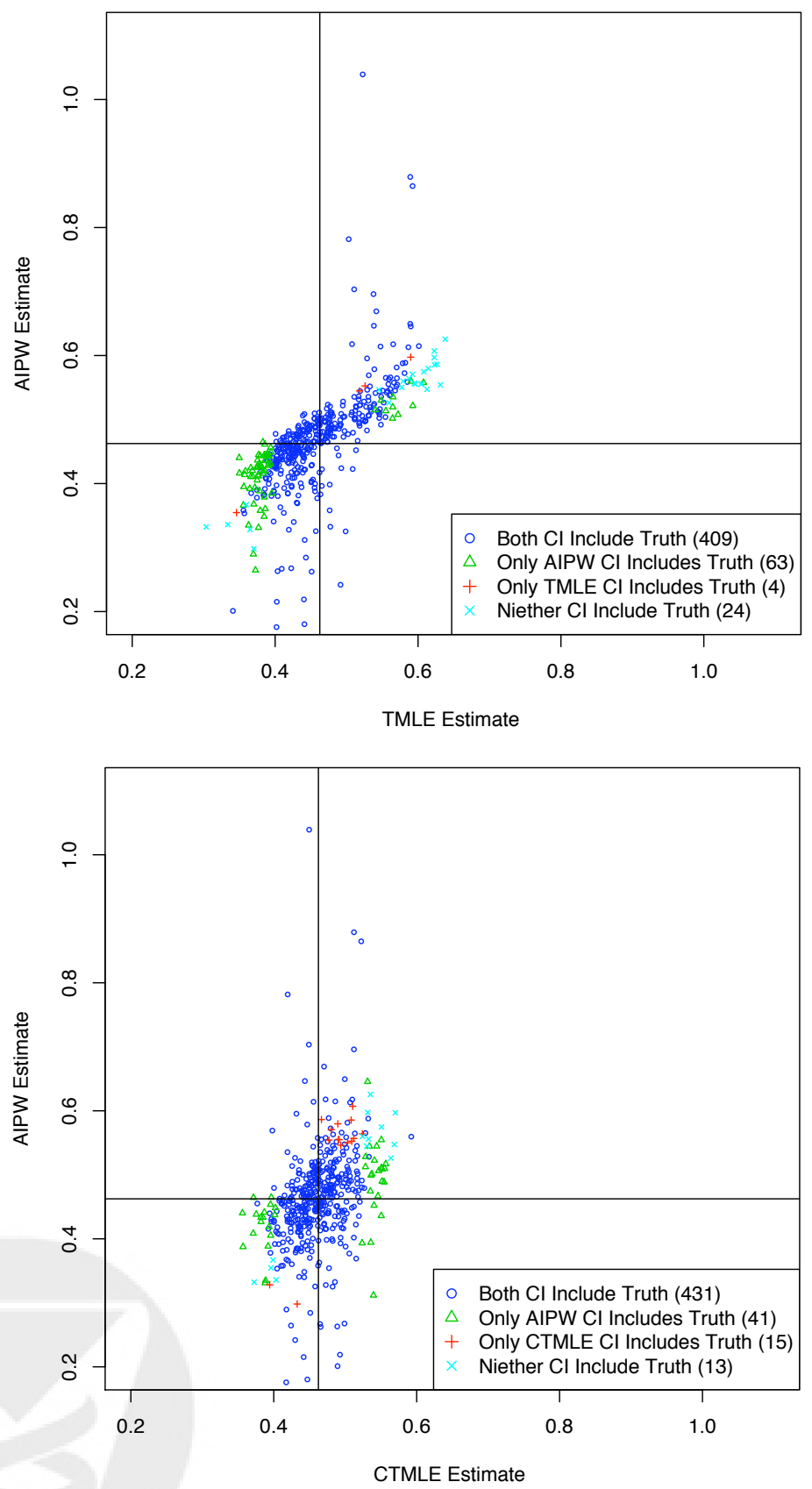

Figure 2: Scatter Plots Of Estimates From High ETA Violation Scenario With Mis-specified Q. 

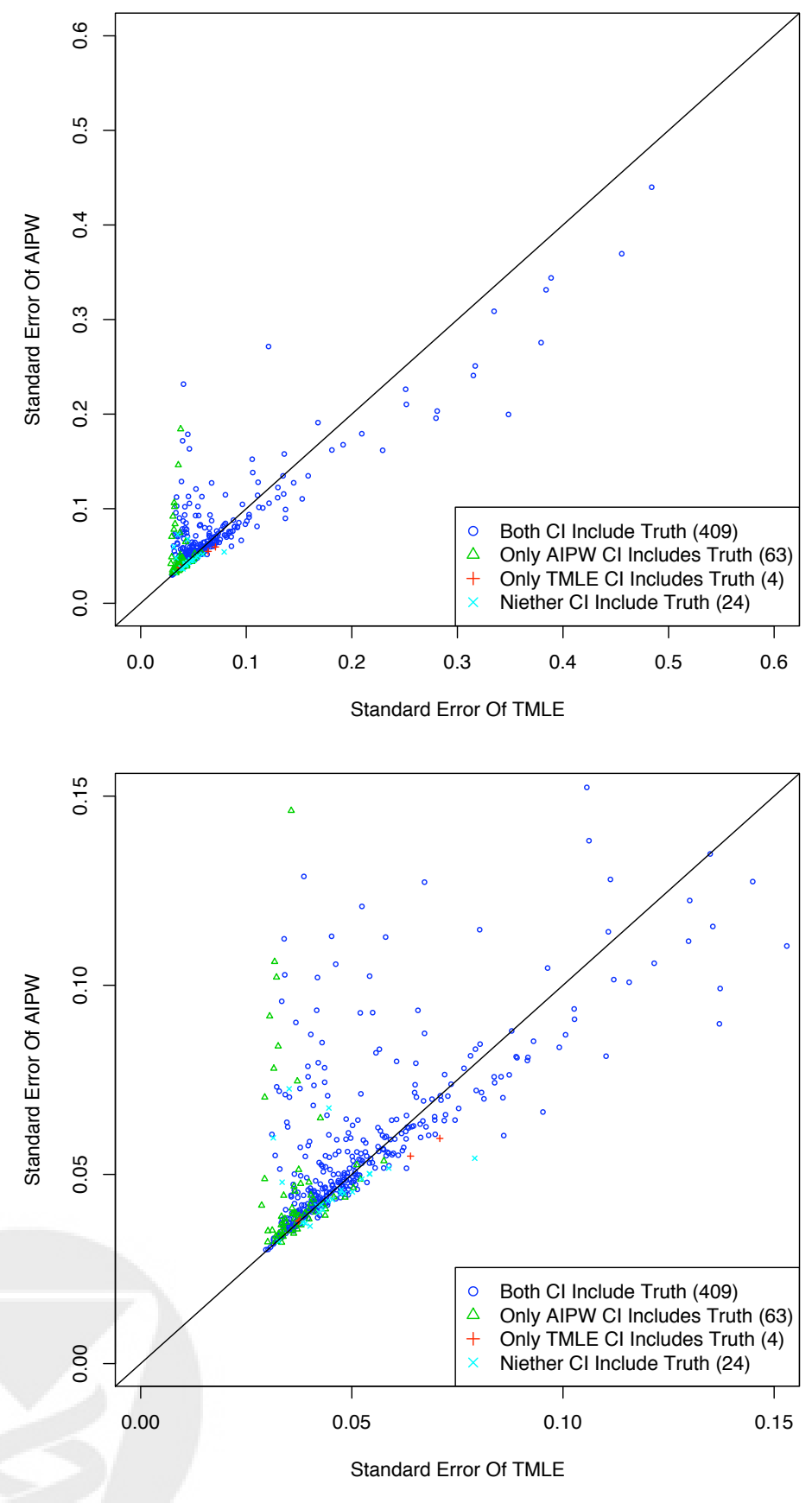

Figure 3: Scatter Plots Of Influence Curve Estimated Standard Errors From High ETA Violation Scenario With Mis-specified Q. 

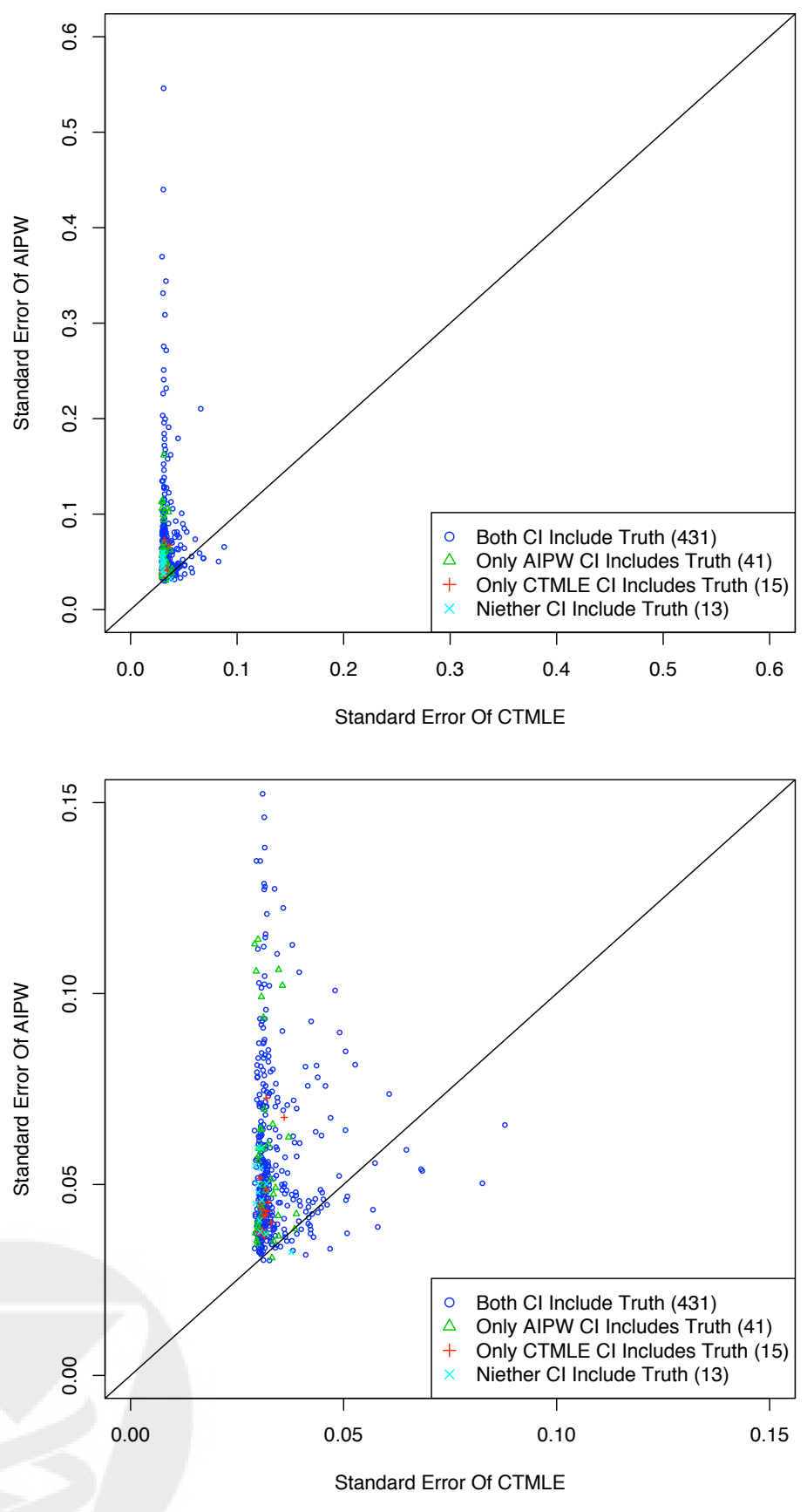

Figure 4: Scatter Plots Of Influence Curve Estimated Standard Errors From High ETA Violation Scenario With Mis-specified Q. 


\begin{tabular}{|c|c|c|c|c|}
\hline \multirow{2}{*}{ Method } & \multicolumn{2}{|c|}{ No ETA } & \multicolumn{2}{|c|}{ "High ETA } \\
\hline & Known Q & Bad Q & Known Q & Bad Q \\
\hline \multicolumn{5}{|l|}{ Coverage Probabilities } \\
\hline TMLE & 0.94 & 0.96 & 0.87 & 0.93 \\
\hline IPW & 0.98 & 0.98 & 0.94 & 0.94 \\
\hline AIPW & 0.94 & 0.95 & 0.94 & 0.92 \\
\hline AIPW w/o ETA variable & 0.94 & 0.95 & 0.94 & 0.38 \\
\hline TMLE w/o ETA variable & 0.94 & 0.96 & 0.94 & 0.38 \\
\hline C-TMLE & 0.94 & 0.94 & 0.94 & 0.82 \\
\hline \multicolumn{5}{|c|}{ Mean Width of Confidence Intervals } \\
\hline TMLE & 0.11 & 0.12 & 0.18 & 0.20 \\
\hline IPW & 0.14 & 0.14 & 0.21 & 0.21 \\
\hline AIPW & 0.11 & 0.12 & 0.17 & 0.25 \\
\hline AIPW w/o ETA variable & 0.11 & 0.12 & 0.12 & 0.12 \\
\hline TMLE w/o ETA variable & 0.11 & 0.12 & 0.12 & 0.12 \\
\hline C-TMLE & 0.11 & 0.12 & 0.11 & 0.15 \\
\hline
\end{tabular}

Table 11: Simulation Results: 95 Percent Wald-Type Confidence Interval Coverage Probabilities And Mean Width Of Confidence Intervals. Variable Causing Violation In ETA Is A Confounder.

fact that there are identifiability concerns in observational data and addresses these issues uniformly, rather than handling them on a case by case basis, or ignoring them completely. The C-TMLE algorithm accomplishes this by using a targeted penalized loss function to make smart choices in determining what variables to adjust for in the estimate of $g$ and only adjusts for variables which have not been fully adjusted for in the initial estimate of $Q$. This allows the C-TMLE estimates to exhibit superefficiency and behave almost as well as the G-comp estimates when the model for $Q$ is specified correctly. In addition, when $Q$ is not specified correctly, the C-TMLE algorithm adjusts in the secondary step only for the variables which improve the estimate of the parameter of interest by considering the bias variance tradeoff for each adjustment. These decisions are always made with respect to how they effect the estimate of the parameter of interest and are not dependent on a loss function designed for the prediction of the treatment/censoring mechanism itself, as it is in the other methods presented. By ignoring the effect of each adjustment on the estimate of the parameter of interest, the other methods have been shown to be highly unstable in finite samples. Furthermore, the C-TMLE is a substitution estimator and obeys the proper bounds of the true model contributing to its overall stability. Lastly, by acknowledging that C-TMLE is a substitution estimator, the bootstrap provides a method to construct valid 95 


\begin{tabular}{lcc}
\hline & Coverage Probabilities & Mean C.I. Widths \\
\hline Quantile Bootstrap & 0.94 & 0.21 \\
Wald Bootstrap & 0.88 & 0.22 \\
Wald Influence Curve & 0.87 & 0.26 \\
\hline
\end{tabular}

Table 12: Simulation Results: Comparison Of Bootstrap And Influence Curve Based Confidence Intervals.

percent confidence intervals that are tighter than the intervals produced by other methods.

\section{References}

[1] A. Hubbard, M.J. van der Laan, and J.M. Robins. Nonparametric locally efficient estimation of the treatment specific survival distributions with right censored data and covariates in observational studies. In D. Berry E. Halloran, editor, Statistical Models in Epidemiology: The Environment and Clinical Trials, pages 135-178. Springer-Verlag, NY, 1999.

[2] K.L. Moore and M.J. van der Laan. Application of time-to-event methods in the assessment of safety in clinical trials. In Karl E. Peace, editor, in Design, Summarization, Analysis 8 Interpretation of Clinical Trials with Time-to-Event Endpoints. Chapman and Hall, 2009.

[3] R. Neugebauer and M.J. van der Laan. Why prefer double robust estimates. Journal of Statistical Planning and Inference, 129(1-2):405-26, 2005.

[4] J. Pearl. Causality: Models, Reasoning, and Inference. Cambridge University Press, Cambridge, 2000.

[5] J.M. Robins and A. Rotnitzky. Recovery of information and adjustment for dependent censoring using surrogate markers. In AIDS Epidemiology, Methodological issues. Bikhäuser, 1992.

[6] M.J. van der Laan and S. Dudoit. Unified cross-validation methodology for selection among estimators and a general cross-validated adaptive epsilon-net estimator: Finite sample oracle inequalities and examples. Technical report, Division of Biostatistics, University of California, Berkeley, November 2003.

[7] M.J. van der Laan, S. Dudoit, and S. Keles. Asymptotic optimality of likelihood-based cross-validation. Statistical Applications in Genetics and Molecular Biology, 3, 2004.

[8] M.J. van der Laan and S. Gruber. Collaborative double robust penalized targeted maximum likelihood estimation. The International Journal of Biostatistics, 2009. 
[9] M.J. van der Laan, E. Polley, and A. Hubbard. Super learner. Statistical Applications in Genetics and Molecular Biology, 6(25), 2007.

[10] M.J. van der Laan and J.M. Robins. Unified methods for censored longitudinal data and causality. Springer, New York, 2003.

[11] M.J. van der Laan and D. Rubin. Targeted maximum likelihood learning. The International Journal of Biostatistics, 2(1), 2006. 